\title{
Cholinergic dysfunction in the dorsal striatum promotes habit formation and maladaptive eating
}

\author{
Mathieu Favier, ${ }^{1,2}$ Helena Janickova, ${ }^{2}$ Damian Justo, ${ }^{3}$ Ornela Kljakic, ${ }^{2}$ Léonie Runtz, ${ }^{1}$ Joman Y. Natsheh, ${ }^{4,5}$ Tharick A. Pascoal, ${ }^{1}$ \\ Jurgen Germann, ${ }^{6}$ Daniel Gallino, ${ }^{6}$ Jun-II Kang, ${ }^{1}$ Xiang Qi Meng, ${ }^{1}$ Christina Antinora, ${ }^{1}$ Sanda Raulic, ${ }^{2}$ Jacob P.R. Jacobsen, ${ }^{7}$ Luc Moquin, ${ }^{1}$ \\ Erika Vigneault, ${ }^{1}$ Alain Gratton, ${ }^{1}$ Marc G. Caron, ${ }^{7,8,9}$ Philibert Duriez, ${ }^{3}$ Mark P. Brandon, ${ }^{1}$ Pedro Rosa Neto, ${ }^{1}$ M. Mallar Chakravarty, ${ }^{6,10,11}$ \\ Mohammad M. Herzallah, ${ }^{4,12}$ Philip Gorwood, ${ }^{3}$ Marco A.M. Prado, ${ }^{2}$ Vania F. Prado, ${ }^{2}$ and Salah El Mestikawy ${ }^{1,13}$ \\ 'Douglas Mental Health University Institute, Department of Psychiatry, McGill University, Montreal, Quebec, Canada. ${ }^{2}$ Robarts Research Institute, Department of Physiology and Pharmacology and \\ Department of Anatomy and Cell Biology, Schulich School of Medicine \& Dentistry, The University of Western Ontario, London, Ontario, Canada. ${ }^{3} \mathrm{GHU}$ Paris Psychiatrie et Neurosciences (CMME, Hospital \\ Sainte-Anne), Institute of Psychiatry and Neuroscience of Paris (INSERM UMR1266), Paris, France. ${ }^{4}$ Palestinian Neuroscience Initiative, Al-Quds University, Abu Dis, Jerusalem, Palestine. ${ }^{5}$ Kessler \\ Foundation, East Hanover, New Jersey, USA. ${ }^{6}$ Cerebral Imaging Centre, Douglas Mental Health University Institute, McGill University, Montreal, Quebec, Canada. ${ }^{7}$ Department of Cell Biology and ${ }^{8}$ Department \\ of Medicine, Duke University, Durham, North Carolina, USA. ${ }^{9}$ Department of Neurobiology, Duke University Medical Center, Durham, North Carolina, USA. ${ }^{10}$ Department of Biological and Biomedical \\ Engineering and "'Department of Psychiatry, McGill University, Montreal, Quebec, Canada. ${ }^{12}$ Center for Molecular and Behavioral Neuroscience, School of Arts \& Sciences-Newark Rutgers University, Newark, \\ New Jersey, USA. ${ }^{13}$ Sorbonne Universités, Université Pierre et Marie Curie UM 119 - CNRS UMR 8246 - INSERM U1130, Neurosciences Paris Seine - Institut de Biologie Paris Seine, Paris, France.
}

\begin{abstract}
Dysregulation of habit formation has been recently proposed as pivotal to eating disorders. Here, we report that a subset of patients suffering from restrictive anorexia nervosa have enhanced habit formation compared with healthy controls. Habit formation is modulated by striatal cholinergic interneurons. These interneurons express vesicular transporters for acetylcholine (VAChT) and glutamate (VCLUT3) and use acetylcholine/glutamate cotransmission to regulate striatal functions. Using mice with genetically silenced VAChT (VAChT conditional KO, VAChTcKO) or VGLUT3 (VGLUT3cKO), we investigated the roles that acetylcholine and glutamate released by cholinergic interneurons play in habit formation and maladaptive eating. Silencing glutamate favored goal-directed behaviors and had no impact on eating behavior. In contrast, VAChTCKO mice were more prone to habits and maladaptive eating. Specific deletion of VAChT in the dorsomedial striatum of adult mice was sufficient to phenocopy maladaptive eating behaviors of VAChTcKO mice. Interestingly, VAChTcKO mice had reduced dopamine release in the dorsomedial striatum but not in the dorsolateral striatum. The dysfunctional eating behavior of VAChTcKO mice was alleviated by donepezil and by L-DOPA, confirming an acetylcholine/dopamine deficit. Our study reveals that loss of acetylcholine leads to a dopamine imbalance in striatal compartments, thereby promoting habits and vulnerability to maladaptive eating in mice.
\end{abstract}

\section{Introduction}

Eating disorders such as anorexia nervosa, bulimia nervosa, and binge eating disorder are severe psychiatric illnesses that are difficult to treat (1). Anorexia nervosa, in particular, has the highest mortality rate of all mental disorders (2). Anorexia nervosa includes persistent restriction of food intake (restrictive-only) and binge eating followed by purging. In contrast, bulimia nervosa and binge eating disorder involve the consumption of a large amount of food in a short period of time, accompanied by a loss of control over eating (3). Currently, there is an unmet need to develop specific treatments for eating disorders.

Eating disorders are attributed to a combination of genetic, neurobiological, psychological, and socio-educational factors (1,

Authorship note: MF and $\mathrm{HJ}$ are co-first authors and contributed equally to this work MAMP, VFP, and SEM contributed equally to this work.

Conflict of interest: The authors have declared that no conflict of interest exists. Copyright: (c) 2020, American Society for Clinical Investigation.

Submitted: March 30, 2020; Accepted: September 2, 2020; Published: November 9, 2020. Reference information: J Clin Invest. 2020;130(12):6616-6630.

https://doi.org/10.1172/JCl138532.
4). Eating disorders are frequently associated with a lack of cognitive flexibility, aberrant reward processing, or obsessive-compulsive disorder $(5,6)$. The inability to interrupt a behavior despite negative consequences suggests a common basis with compulsive disorders (7). An imbalance between goal-directed behaviors and habitual behaviors has been suspected to be a central cause of eating disorders $(8,9)$.

Goal-directed behaviors are observed when individuals are engaged in reaching a specific objective. Hence, action selection is governed by its outcome. Habits emerge from goal-directed learning after behaviors become automated as a result of repetition. Habits are driven by environmental stimuli and are independent from outcomes (10).

The dorsal striatum is a key hub for the regulation of goaldirected behaviors and habits (10). While the dorsomedial striatum (caudate in humans) is crucial for the acquisition of flexible goal-directed behaviors, the dorsolateral striatum (putamen in humans) modulates rigid habitual behaviors. Dopaminergic (DA) transmission is a pivotal regulator of the transition from goaldirected behaviors to habits $(11,12)$. 
Phase 1 - Instrumental learning

A

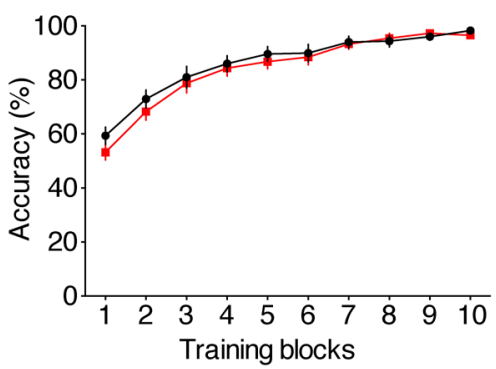

B

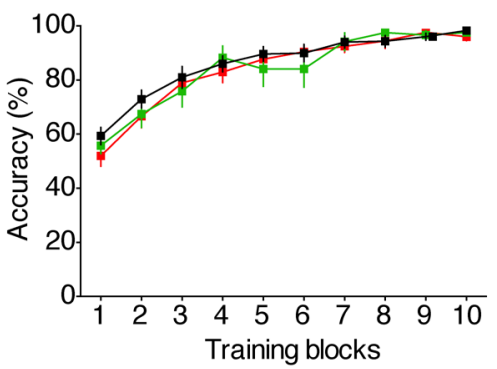

Phase 2 - Outcome devaluation

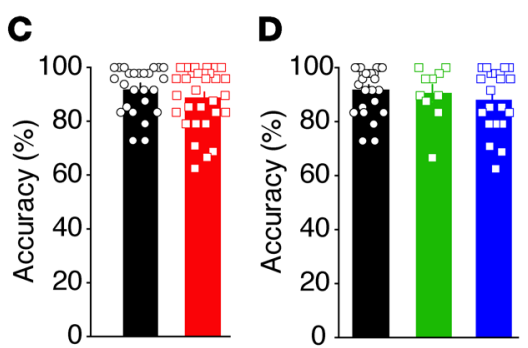

$\mathbf{E}$

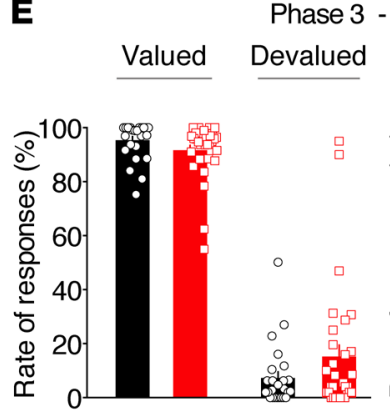

- "Slip of action"

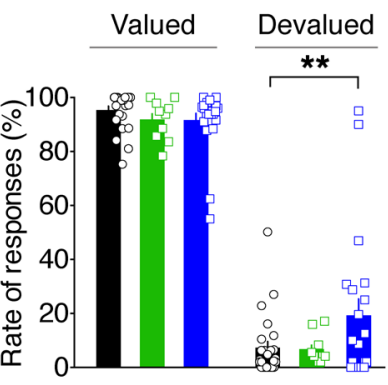

$\mathbf{F}$

Phase 4 - Inhibitory control

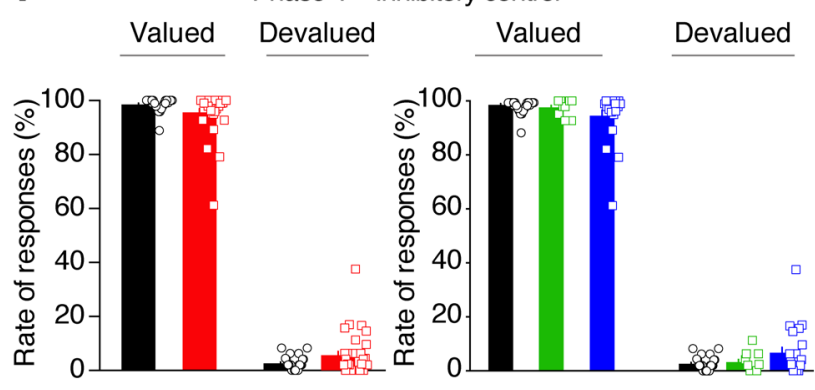

G

H

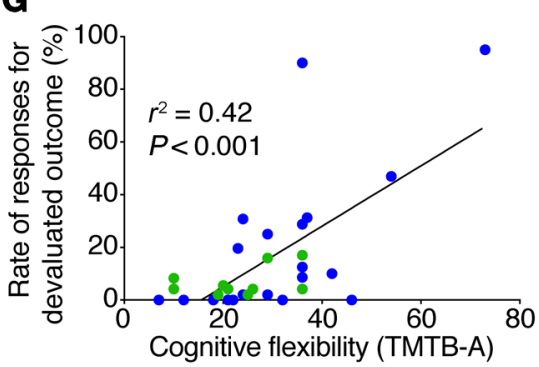

Ctrl $(n=25)$

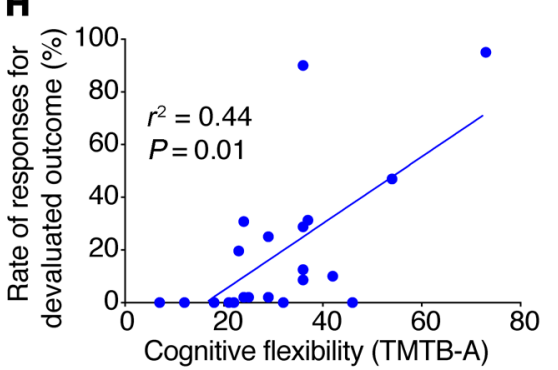

Patients (31) $\quad \square \rightarrow \quad$ AN-R $(n=21)$

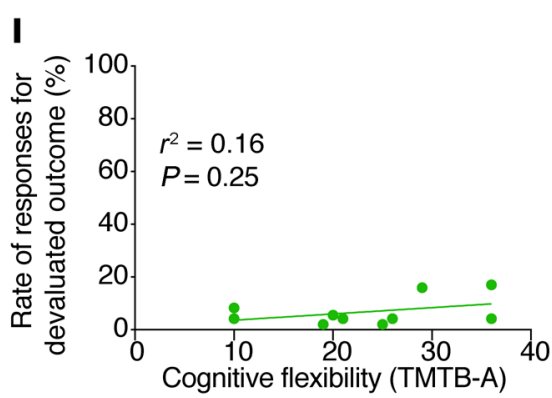

$\operatorname{ED}-B P(n=10) \quad \square \square$

Figure 1. The balance between goal-directed behaviors and habits is altered in a subgroup of restrictive-type anorexia nervosa patients. (A and $B$ ) Instrumental learning stage. Eating disorder (ED) patients showed similar instrumental learning performances compared with healthy controls (HCs); accuracy was increased across blocks of trainings for all groups. (C and D) Outcome devaluation stage. In the second phase, accuracy of ED patients to direct their choices toward still-valued outcomes was not modified in comparison with HCs, suggesting that knowledge of response-outcome associations was not affected by the pathology. (E) Slip-of-action stage. During phase 3, the percentage of responses for valued outcome was not modified for ED patients. Together, these data suggest that ED patients performed similarly to controls in response to devalued outcome, but performance of ED patients was highly heterogeneous compared with controls. Dividing between restrictive anorexia nervosa (AN-R) and binge eating/purging ED patients (ED-BP) revealed that the AN-R subgroup responded significantly more to devalued items in phase 3. (F) Baseline stage of inhibitory control. No difference was found between $\mathrm{HCs}, \mathrm{AN}-\mathrm{R}$, and ED-BP in the percentage of responses to valued or devalued stimuli. (G-I) Correlation analyses between cognitive flexibility as evaluated by the Trail Making Test B-A (TMTB-A) and the percentage of responses to devalued outcome measured in phase 3 . (C) The rate of responses for devalued outcome of ED patients was correlated with a deficit of cognitive flexibility. (H and I) This correlation was specific to the AN-R group (H) but was not seen with the ED-BP subgroup (I). Two-way repeated-measures ANOVA (A and $\mathbf{B}$ ) and post hoc comparison with the method of contrasts; unpaired 2-tailed $t$ test (C); 1-way ANOVA (D); 2-way ANOVA (E and F) and post hoc comparison with Dunnett's test. Dimensional analyses were performed by parametric simple linear regressions for $\mathbf{G}-\mathbf{I}$. ${ }^{* *} P<0.01$.

Cholinergic interneurons (ChIs) from the striatum are also major regulators of striatal functions and exert complex effects over DA efflux $(13,14)$. ChIs express both the vesicular acetylcholine transporter (VAChT; Slc18a3) and the atypical vesicular glutamate transporter type 3 (VGLUT3; Slc17a8) (15). Thus, ChIs release both acetylcholine (ACh) and glutamate (Glu) to regulate striatal functions $(16,17)$. ACh and Glu released from ChIs differentially affect DA transmission in the ventral striatum and reward-guided behaviors $(13,18)$. Interestingly, rodents with selective ablation of ChIs present deficits of behavioral flexibility and increased compulsive behavior $(19,20)$. However, how each individual neurotransmitter (ACh or Glu) released by ChIs affects the transition between goal-directed behaviors and habits, and therefore potentially influences maladaptive eating, is still obscure $(21,22)$.

Using a computer-based neurocognitive task to evaluate the balance between goal-directed behaviors and habits in humans $(23,24)$, we here confirm that a subgroup of patients with restrictive anorexia nervosa are more prone to habitual behaviors than healthy controls. Using mouse models, we show that disrupting $\mathrm{ACh} / \mathrm{Glu}$ cotransmission, by genetically inactivating VAChT 
(VAChT-conditional KO mice, VAChTcKO mice), but not VGLUT3 (VGLUT3cKO mice), impairs behavioral flexibility, promotes habit formation, and consequently causes mice to be more susceptible to maladaptive eating. Specific deletion of VAChT from the dorsomedial striatum is sufficient to phenocopy the maladaptive eating of VAChTcKO mice. In parallel, we observed that DA efflux decreases in the dorsomedial striatum but remains unchanged in the dorsolateral striatum of VAChTcKO mice. This observation points to the possibility that uneven DA transmission in striatal compartments could underlie excessive habit formation and maladaptive eating. Indeed, increasing extracellular ACh with donepezil (an acetylcholine esterase inhibitor) or DA efflux with L-DOPA abolishes the self-starvation phenotype of VAChTcKO mice in the activity-based anorexia model.

Our experiments reveal mechanisms underlying vulnerability to maladaptive eating and identify striatal ACh/Glu cotransmission as a potential target to correct excessive habitual behavior and pathological eating.

\section{Results}

Enhanced habit formation in restrictive-type anorexic patients. Whether patients with eating disorders have a dysregulated balance of goal-directed behaviors and habits is not clearly established $(9,25)$. Thus, we investigated habit formation in a group of patients with eating disorders using a computer-based neurocognitive task $(24,25)$. These experiments were conducted with a cohort of 25 healthy controls and 31 patients with eating disorders (Supplemental Table 1; supplemental material available online with this article; https://doi.org/10.1172/JCI138532DS1). Patients with eating disorders were divided in 2 categories: restrictive-type anorexia patients (AN-R, $n=21$ ) and binge eating/purging eating disorder patients (ED-BP, $n=10$ ). During the learning phase, accuracy increased over training sessions, and instrumental learning performances were similar across the 3 groups of subjects (Figure $1, \mathrm{~A}$ and B). During the initial phase, reaction time decreased across blocks of training and was not altered in patients with eating disorders (Supplemental Figure 1, A and B). In the second phase, participants were tested on their knowledge of response-outcome associations learned during the first stage. In phase 2, no differences were observed in response accuracy (Figure 1, C and D) or reaction time (Supplemental Figure 1, C and D) among groups. During the "slip-of-action" stage (phase 3), the percentage of responses was not significantly different between controls and patients for valued items or devalued items when eating disorder patients were considered altogether (Figure 1E). However, performance of the AN-R and ED-BP subgroups of patients was heterogeneous compared with that of healthy controls (Welch's test, $P=0.01$, and Brown-Forsythe test, $P=0.02$; Supplemental Table 2). Some individuals suffering from AN-R persistently responded to stimuli associated with devalued outcomes (Figure 1E), while ED-BP patients responded similarly to controls (Figure 1E). This finding suggests that a subgroup of patients with restrictive-type anorexia was not able to withhold inappropriate responses for devalued outcomes, indicating a shift toward habitual behavior. Finally, in phase 4 , inhibitory control was tested. During this phase, we found no significant differences between controls and patients with AN-R or ED-BP in responses to valued or devalued stimuli (Fig- ure $1 F)$. Hence, the habit bias observed during the slip-of-action stage in AN-R patients was not due to a deficit in response inhibition. Interestingly, the rate of responses for devalued outcome was positively correlated with the body mass index (BMI) in AN-R patients (Supplemental Figure 1, E-G). In contrast, neither Hospital Anxiety and Depression Scale score nor disease duration was correlated with the rate of devalued outcome responses (Supplemental Figure 1, H and I). Furthermore, in AN-R patients, but not ED-BP patients, the percentage of response to devalued outcomes was correlated with a lack of cognitive flexibility (measured with the Trail Making Test B minus A; Figure 1, G-I).

Women constituted the vast majority of healthy controls (19 of 25) and patients (29 of 31). This sex difference had only a small impact, as the global effect was close to the one observed when the cohort was restricted to women. In both cases, we found a significant interaction between condition (value) and group performance during phase 3 of the test.

Altogether, these results show that a subgroup of anorexic patients presents a disruption in the balance between goal-directed behaviors and habits, which is correlated with a lack of cognitive flexibility.

VAChT-mutant mice have an impairment in flexibility and are more prone to habit formation. The dorsal striatum plays a pivotal role in the regulation of the balance between flexible goal-directed behaviors and inflexible habits $(10,26,27)$. The striatal network, and its cognitive function, are powerfully regulated by ChIs (19, 28). However, how striatal functions are influenced by ACh or Glu cotransmission is not yet established. To specifically address the roles of ACh and Glu released by ChIs in behavioral flexibility, habit formation, and maladaptive eating, we used genetically modified mice in which VAChT or VGLUT3 was deleted selectively in ChIs using the Cre-Lox recombination approach. This method allowed the deletion of either VAChT (D2 driver) or VGLUT3 (ChAT or D2 driver) from ChIs (respectively, VAChTcKO mice, VGLUT3cKO mice, or VGLUT3cKO-D2Cre mice). Extensive anatomical characterization of mutant mice was performed (Supplemental Figures 2 and 3). Since the highest density of D2R expression in ChIs is found in the dorsal striatum of rodents $(29,30)$, the efficiency of D2-Cre-Lox deletion was maximal in the dorsal striatum of VAChTcKO mice (Supplemental Figure 2E). Extracellular ACh was dramatically reduced in the dorsal striatum of VAChTcKO mice (Supplemental Figure 2G). Silencing of VGLUT3 by D2-Cre or ChAT-Cre drivers was also maximal in the dorsal striatum (Supplemental Figures 2 and 3).

Behavioral flexibility allows for the adjustment of a behavioral response to unexpected conditions. Lack of behavioral flexibility is a well-established marker of eating disorders (6). We used a touchscreen pairwise visual discrimination and reversal learning task to explore behavioral flexibility in VGLUT3cKO mice and VAChTcKO mice. In the acquisition phase, mice were taught to discriminate and choose a rewarded over an unrewarded image (Figure $2 \mathrm{~A}$ ). After reaching the criterion ( $80 \%$ correct responses out of 30 trials in 2 consecutive sessions), the contingency was switched (Figure 2D), and mice had to extinguish the previous response and learn the new rewarded association. Both VGLUT3cKO mice and VAChTcKO mice did not differ from controls during the acquisition phase, indicating that learning was not significantly affected by 
A
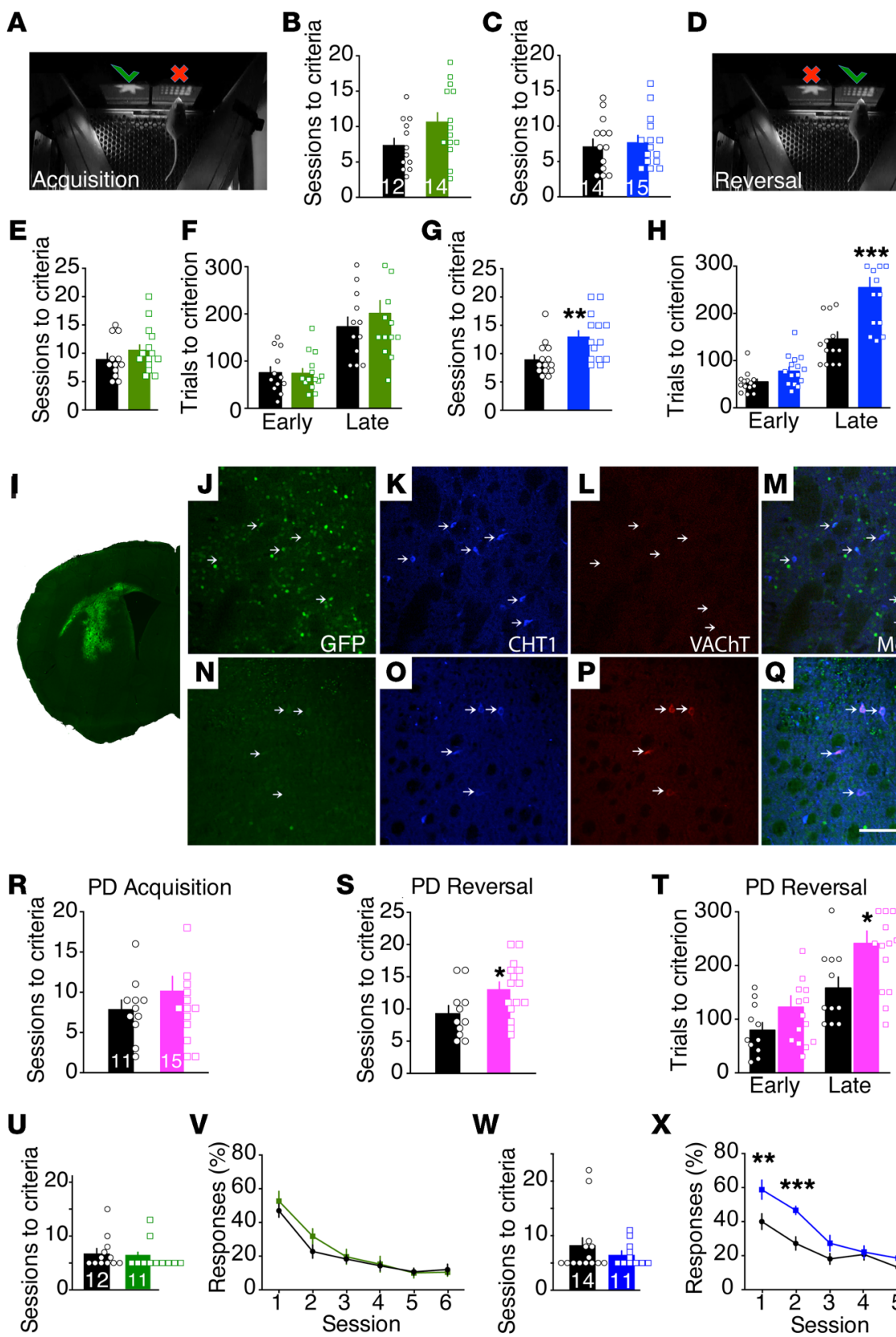

$\mathbf{X}$

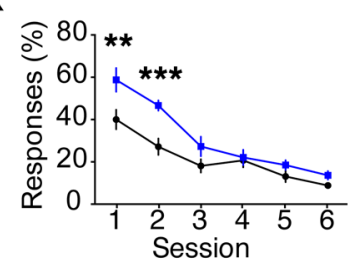

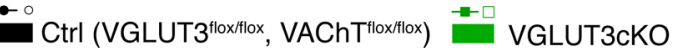

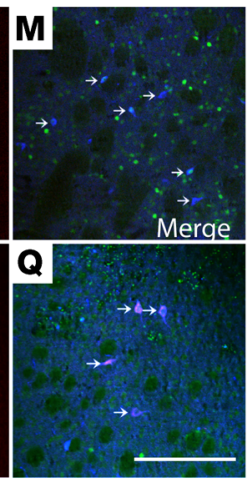

D

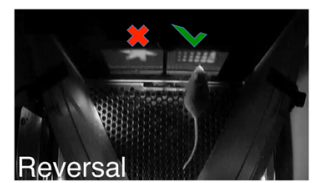

Figure 2. Acetylcholine or glutamate released from striatal cholinergic interneurons differentially regulates behavioral flexibility. (A-D) Number of sessions needed for VCLUT3CKO mice $(n=14)$, VAChTcKO mice $(n=15)$, and controls ( $n=12$ and 14 , respectively) to reach learning criterion. (E-H) Performance of mice during reversal learning. ( $\boldsymbol{E}$ and $\mathbf{G}$ ) Number of sessions to criterion. ( $\mathbf{F}$ and $\mathbf{H}$ ) Number of trials during early (performance is below chance) and late (performance is equal to or above chance) phases of reversal training. (I-Q) Immunofluorescence analysis of AAV-Cre-GFP expression in the dorsal striatum of VAChT ${ }^{\mathrm{fl} / \mathrm{fl}}$ mice. Brain sections immunostained for GFP (green, I), choline transporter (CHT1; blue, $\mathbf{K}, \mathbf{M}, \mathbf{0}$, and $\mathbf{Q})$, and VAChT (red, $\mathbf{L}, \mathbf{M}, \mathbf{P}$, and $\mathbf{Q})$. (M and $\mathbf{Q}$ ) Merged images. (J-M) Images from AAV-CreGFP-expressing area. (N-Q) Images from a striatal area lacking GFP-Cre expression. Arrows indicate cholinergic interneurons not expressing (L) or expressing VAChT (P). Scale bar: $200 \mu \mathrm{m}$. (R-T) Performance of VAChTcKO-DS mice. (R) Number of sessions required for VAChTcKODS mice $(n=15)$ and controls $(n=11)$ to reach criterion during acquisition phase. ( $\mathbf{S}$ and $\mathbf{T}$ ) Performance in the reversal phase of the task. (S) Number of sessions required for mice to reach criterion. (T) Number of trials during early and late phases of reversal. (U-X) Extinction tests were performed in mice previously trained in other touchscreen tasks. Number of training sessions required for VGLUT3CKO mice (U) or VAChTcKO mice (W) to learn the task. ( $\mathbf{V}$ and $\mathbf{X )}$ Rate of responses during extinction phase. Unpaired 2-tailed $t$ test (B, C, E, G, R, and $\mathbf{S}$ ); 2-way ANOVA (F, $\mathbf{H}, \mathbf{T}, \mathbf{V}$, and $\mathbf{X})$ and Bonferroni's post hoc comparison. ${ }^{*} P<0.05$, ${ }^{* *} P<$ 0.01 , ${ }^{* *} P<0.001$. All data are mean $\pm \mathrm{SEM}$. ablation of either VGLUT3 or VAChT (Figure 2, B and C). During reversal learning, performance of VGLUT3cKO mice was also similar to that of controls (Figure 2, E and F; and Supplemental Figure 4), indicating that these mutants were able to inhibit the previously learned association and acquire a new contingency. In contrast, VAChTcKO mice needed significantly more sessions to reach criterion on reversal learning in comparison with controls (Figure 2G). Further analysis of reversal learning sessions indicated that in the early phase (sessions with $<50 \%$ correct responses), VAChTcKO mice and controls were equally able to quickly stop responding to the previously correct choice (Figure $2 \mathrm{H}$, Early), indicating that perseverative errors for both genotypes were comparable. In contrast, in the late phase of the task (sessions with $>50 \%$ correct responses), the number of incorrect choices (regressive errors) made by VAChTcKO mice was significantly higher in comparison with controls, indicating that VAChTcKO mice have an impaired ability to maintain a new correct choice pattern. That is, after learning an action-outcome association, VAChTcKO mice have difficulty in changing it (Figure 2H, Late). A similar deficit was observed in $\mathrm{VAChT}^{\mathrm{f} / \mathrm{l}}$ mice that received bilateral AAV-Cre injection in the dorsal striatum resulting in a limited deletion of VAChT (Figure 2, I-T). Importantly, deletion of VAChT in the striatum did not affect attentional performance or inhibition control determined in the 5-choice serial reaction time task (5-CSRTT) (Supplemental Figure 5, A-C). Moreover, cohorts of VAChTcKO mice and VGLUT3cKO mice that were previously tested in the reversal learning task were also evaluated in extinction tests. VGLUT3cKO mice showed no deficiency in the ability to stop responding (Figure 2, $\mathrm{U}$ and V; and 
A

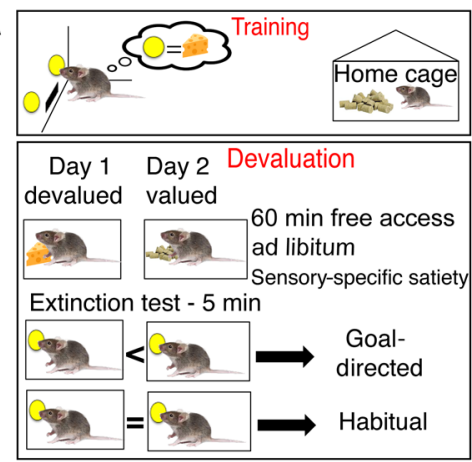

D

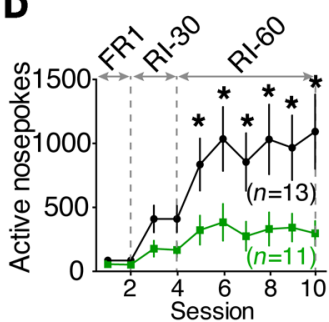

$\mathbf{F}$

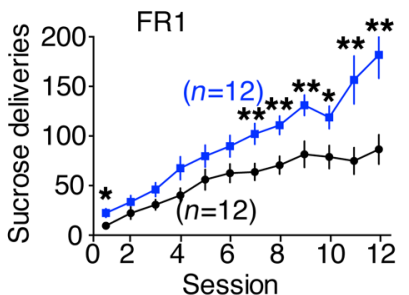

I

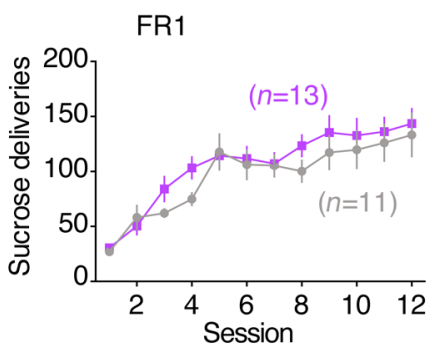

B

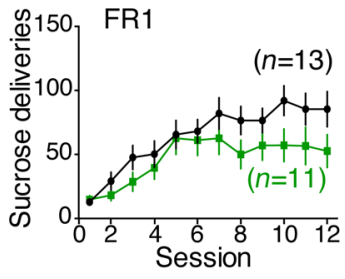

C

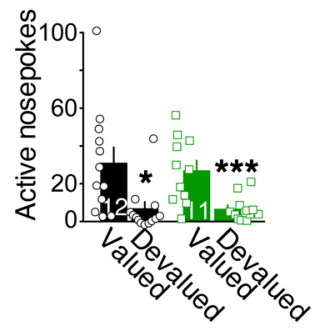

E
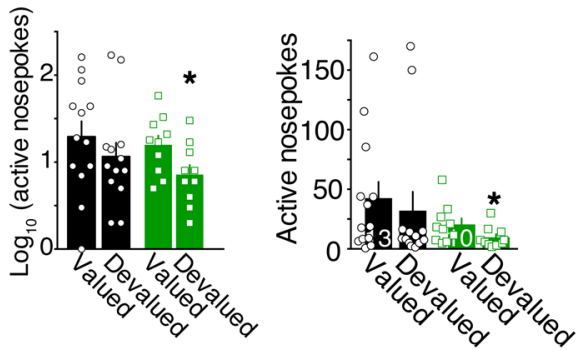

G

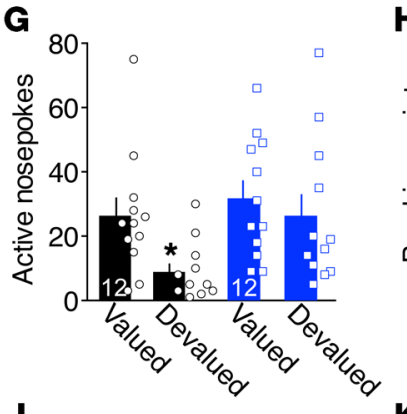

J $\mathbf{K}$ Progressive ratio

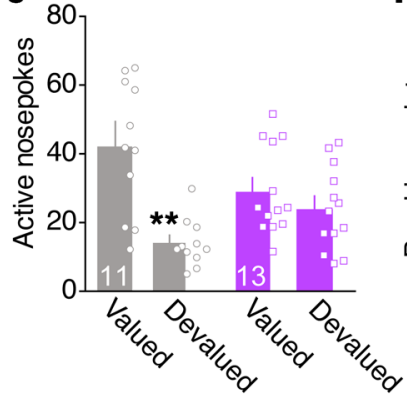

H Progressive ratio
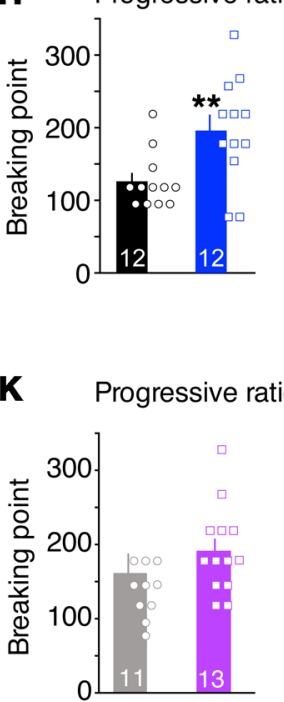

Figure 3. Acetylcholine or glutamate released from striatal cholinergic interneurons differentially regulates transition from goal-directed behaviors to habits. (A) Diagram showing the training for sucrose self-administration and devaluation test. (B) Number of sucrose pellet deliveries for VGLUT3cKO mice $(n=11)$ versus controls $(n=13)$ during FR1 training. (C) Devaluation test for VGLUT3cKO mice $(n=11)$ versus controls $(n=12)$ after FR1 training. (D) Number of sucrose pellet deliveries for VCLUT3cKO mice ( $n=11$ ) versus controls $(n=13)$ during the 2 last sessions of FR1 training, followed by 8 sessions of random interval $(\mathrm{RI})$ training to bias mice toward habits. (E) Devaluation after RI training presented in $\log _{10}$ and in raw number of nosepokes. (F) Number of sucrose pellet deliveries for VAChTcKO male mice $(n=12)$ versus controls ( $n=12$ ) during FR1 training. (G) Devaluation test of VAChTcKO male mice after FR1 training. (H) Progressive ratio (PR) test to assess the motivation of VAChTcKO male mice $(n=12)$ and littermate controls $(n=12)$. (I) Number of sucrose pellet deliveries for VAChTcKO female mice ( $n=$ 13) versus controls $(n=11)$ during FR1 training. (J) Devaluation test of VAChTcKO mice $(n=13)$ versus respective controls $(n=11)$. (K) PR test of VAChTcKO female mice $(n=13)$ and littermate controls $(n=11)$. Two-way repeated-measures ANOVA (B, D, F, and I) and post hoc comparison with the method of contrasts; paired 2-tailed $t$ test (C, E, G, and J); unpaired 2-tailed $t$ test (H and $\mathbf{K})$. ${ }^{*} P<0.05,{ }^{* *} P<0.01,{ }^{* *} P<0.001$.

\footnotetext{
Ctrl (VGLUT3 $3^{\text {floxflox }}$, VAChT $T^{\text {flox/flox }}$ male) $\bullet$ 。

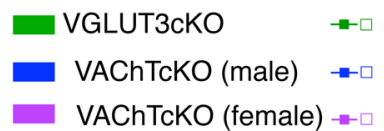

Supplemental Figure 6, A and B, for VGLUT3cKO-D2Cre), whereas VAChTcKO mice presented extinction deficits compared with controls (Figure 2, W and X). Taken together, these data suggest that silencing VGLUT3 and glutamate released by ChIs had no effect on behavioral flexibility. In contrast, reducing VAChT and cholinergic signaling in the dorsal striatum significantly impaired behavioral flexibility and extinction.

VAChT-mutant mice are more prone to habit formation. We then tested whether the behavioral flexibility deficit observed in VAChTcKO mice was associated with an altered transition from goal-directed behaviors to habits. In order to bias mice toward goal-directed behaviors, they were trained for 12 days under continuous reinforcement using a fixed-ratio 1 paradigm (FR1; 1 nosepoke is associated with the delivery of 1 sucrose pellet) (Figure 3A, Training). Then, during the devaluation tests, the outcome was made less desirable by exposure of mice for 1 hour to sucrose pellets (devalued condition) or to regular chow (valued condition) (Figure 3A, Devaluation). A decrease in responding to the devalued condition compared with the valued condition indicates goal-directed behavior, whereas insensitivity to devaluation demonstrates habitual 
A Binge-like sucrose overconsumption

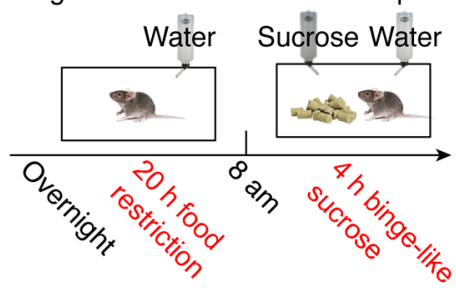

B

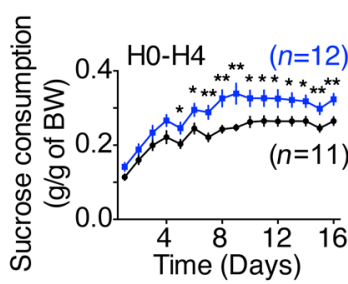

E

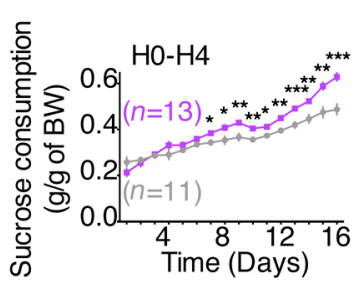

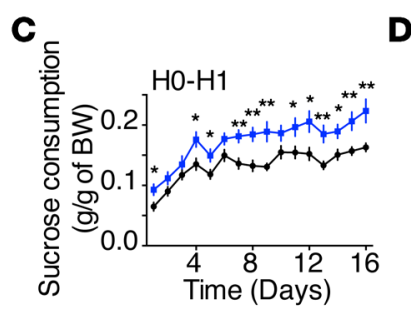
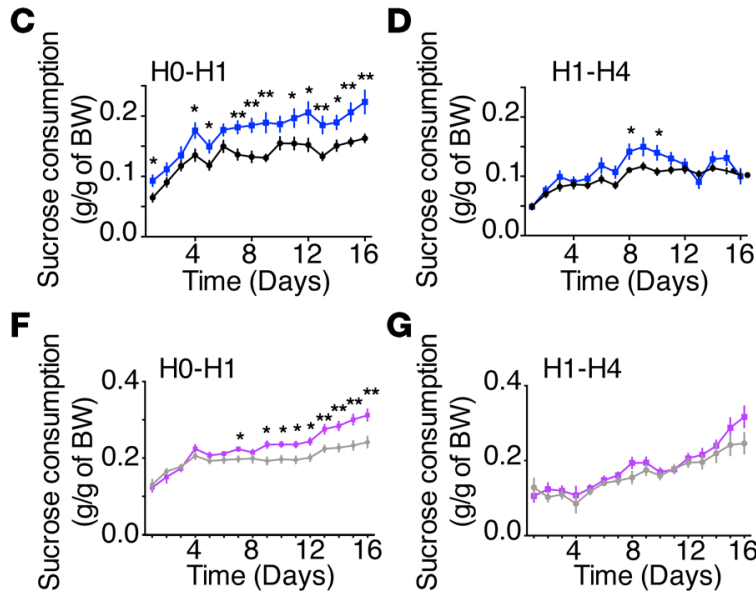

H

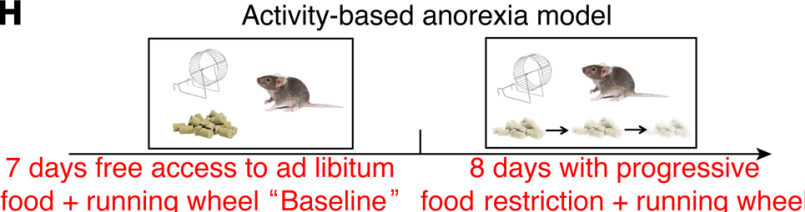

I

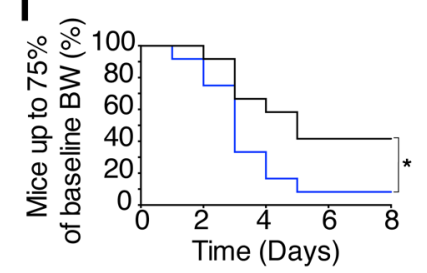

J

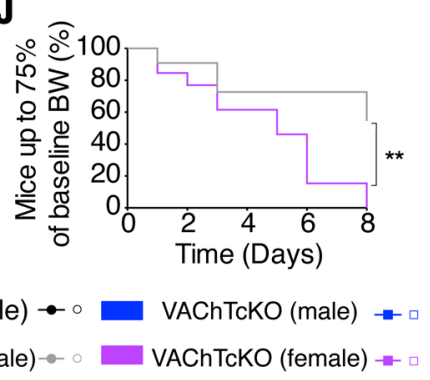

Figure 4. VAChTcKO mice develop binge- and anorexia-like phenotypes. (A) Diagram showing the binge-like sucrose overconsumption model. Mice alternated daily between 20-hour food restriction and 4-hour simultaneous access to food and to a highly concentrated sucrose solution. (B) Daily sucrose consumption by male VAChTcKO mice $(n=12)$ and littermate controls $(n=12)$ during access to sucrose solution (HO-H4). (C) Daily sucrose consumption by male VAChTcKO mice and controls during the first hour of access to sucrose solution (HO-H1). (D) Daily sucrose consumption by male VAChTcKO mice and controls during the last 3 hours of access to sucrose solution (H1-H4). (E) Daily sucrose consumption by female VAChTcKO mice $(n=13)$ and littermate controls ( $n=11)$ during access to sucrose solution ( $\mathrm{HO}-\mathrm{H} 4)$. (F) Daily sucrose consumption by female VAChTcKO mice and littermate controls during the first hour of access to sucrose solution ( $\mathrm{HO}-\mathrm{H1}$ ). (C) Daily sucrose consumption by female VAChTcKO mice and controls during the last 3 hours of access to sucrose solution ( $\mathrm{H} 1-\mathrm{H} 4)$. (H) Diagram of the activity-based anorexia (ABA) model. During baseline, mice were habituated to cages with running wheel for 7 days receiving ad libitum food. Then, mice underwent a progressive time-restricted access to food for 8 days. (I and $\mathbf{J})$ Percentage of male $(\mathbf{I}, n=12)$ and female $(\mathrm{J}, n=12)$ VAChTcKO mice and controls $(n=12)$ that reached a critical level of less than $75 \%$ baseline BW compared with controls during ABA. Note that male and female VAChTcKO mice were significantly more affected than control mice. Two-way repeated-measures ANOVA (B-G) and post hoc comparison with the method of contrasts; Kaplan-Meier test (I and J) and post hoc comparison with log-rank Mantel-Cox and Gehan-Breslow-Wilcoxon tests. ${ }^{*} P<0.05,{ }^{* *} P<0.01,{ }^{* *} P<0.001$.

behavior (31). Because of interindividual differences and variability during baseline training, devaluation results are shown as raw data (number of active nosepokes; Figure 3, C, E, G, and J) as well as normalized data (Supplemental Figure 7, A, D-G, I, and M).

Performance of VGLUT3CKO mice was similar to that of littermate controls during operant instrumental training (Figure 3B). During devaluation tests, both VGLUT3cKO mice and controls performed less in the devalued versus the valued condition (Figure 3C and Supplemental Figure 7B for VGLUT3-constitutive-KO mice), revealing goal-directed behaviors.

Male VAChTcKO mice made significantly more nosepokes compared with controls during the last days of FR1 training (Figure $3 \mathrm{~F}$ ). Conversely, performance of female VAChTcKO mice was similar to that of controls throughout instrumental training (Figure 3I). In the devalued condition, control mice decreased their responding (goal-directed behavior). However, male and female VAChTcKO mice exhibited comparable performance in valued and devalued conditions (Figure 3, G and J; and Supplemental
Figure 7, E-G, for normalized analysis). Since VAChTcKO male mice increased their performance during days 7-12 of FR1 training (as shown in Figure 3F), results of devaluation tests were also normalized to the performance of mice on the last day of FR1 training (Supplemental Figure 7F; normalized results for females are shown in Supplemental Figure 7G). Regardless of the type of analyses that were conducted, male and female VAChTcKO mice consistently performed similarly in valued versus devalued conditions. These findings demonstrate that VAChTcKO mice, unlike VGLUT3cKO mice and controls, switched toward habitual behavior after only 12 days of FR1 training.

Independent cohorts of VGLUT3cKO mice and VAChTcKO mice were trained under continuous reinforcement (FR1) and then switched to random interval (RI) training to bias mice toward habits (31). Mice were trained for 2 days on RI-30 (a pellet can only be earned once every 30 seconds and the point at which lever is activated varies randomly) and then switched to 6 days on RI- 60 (same procedure, except a pellet is earned every 60 seconds). During RI 
A
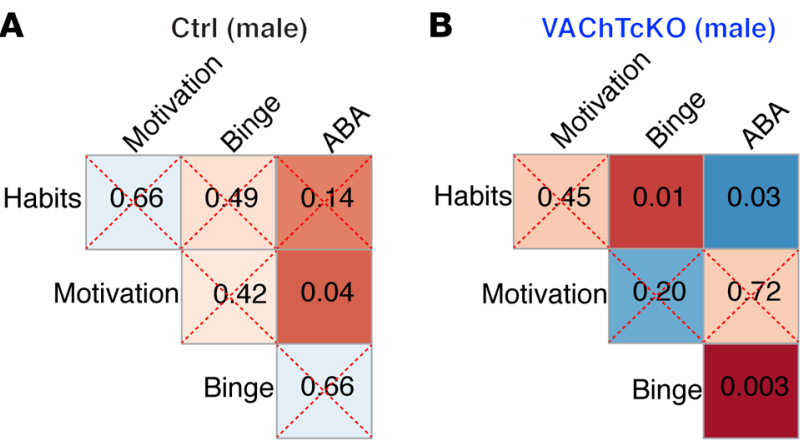

E

Ctrl

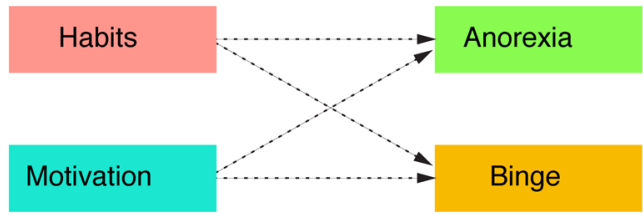

G

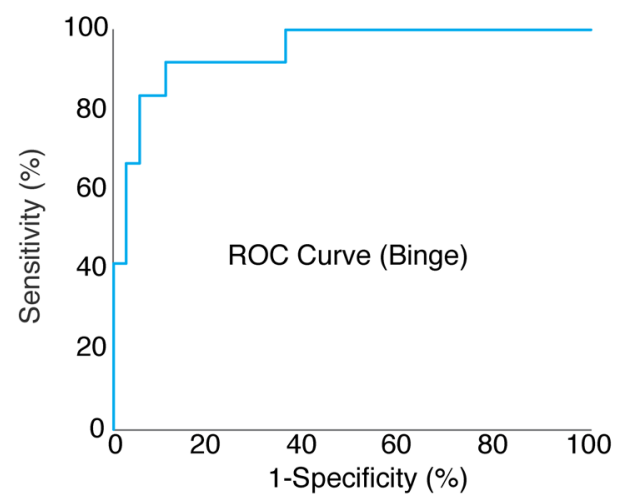

C

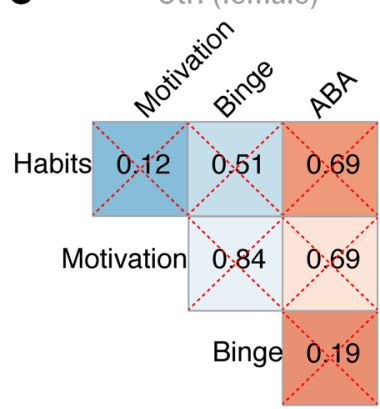

D VAChTcKO (female)

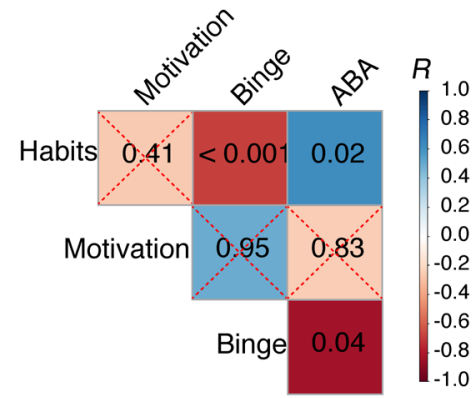

$\mathbf{F}$
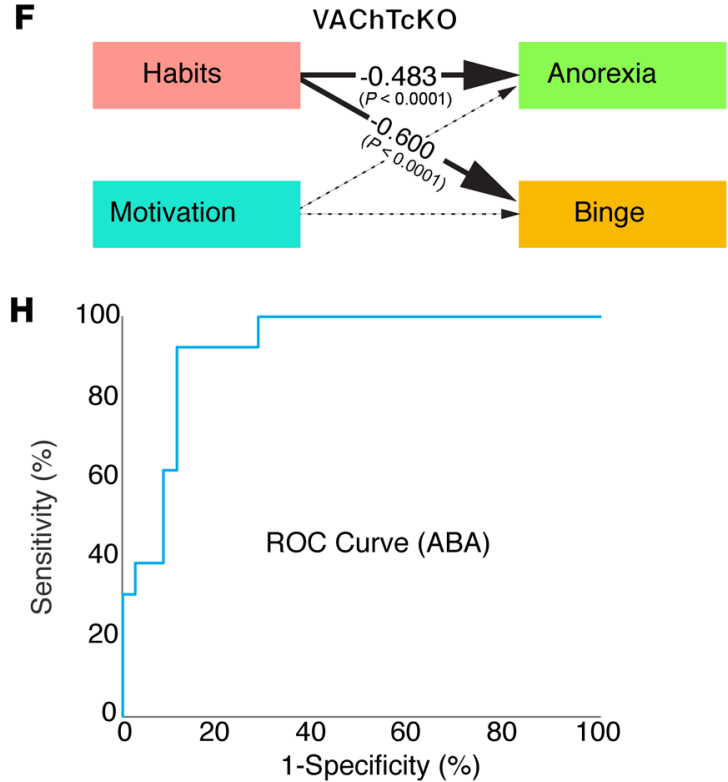

Figure 5. Propensity to habitual behavior predicts binge- and anorexia-like phenotypes in VAChTcKO mice. (A-D) Matrices show $P$ values of the associations between behavioral data in control (Ctrl) male mice (A), VAChTcKO male mice (B), control female mice (C), and VAChTcKO female mice (D). (E and F) Estimates of the structural equation model representing the association between habits (devaluation scores), motivation (breaking point during PR test), anorexia-like phenotype (food intake during ABA model), and binge-like phenotype ( $\mathrm{HO}-\mathrm{H} 1$ sucrose intake during binge-like sucrose overconsumption test) for control mice or VAChTCKO mice. These associations were adjusted by sex. Solid lines represent significant effects, whereas dashed lines represent nonsignificant ones. The effect sizes ( $\beta$ estimates) presented in the figure were fully standardized and therefore may be compared. Estimates were derived from a model that fit the data well (root mean square error of approximation $=0.0$, standardized root mean square residual $=0.00$, comparative fit index $=1.000$, Akaike information criterion $=639.918$, and Bayesian information criterion $=650.888$ ) and show that habits correlate to maladaptive eating only in VAChTcKO mice. (G) ROC curves based on probabilities generated from logistic regression analyses. The logistic regression model that generated these probabilities included valuation scores, devaluation scores, motivation scores, genotype, and sex to predict binge. The logistic regression model showed $83.3 \%$ sensitivity and $94.4 \%$ specificity in predicting vulnerability to binge-like behavior. The significant predictors were the valuation scores and sex. Devaluation scores were approaching significance. The area under the ROC curve (diagnostic power) is $94.4 \%$. (H) The logistic regression model that generated the probabilities included valuation scores, devaluation scores, motivation scores, genotype, and sex to predict anorexia. The model exhibited $76.9 \%$ sensitivity and $88.6 \%$ specificity in identifying vulnerability to anorexia-like behavior. The only significant predictor was the devaluation scores. The area under the ROC curve (diagnostic power) is $92.1 \%$.

training, VGLUT3cKO mice showed decreased nosepoke activity relative to control mice (Figure 3D and Supplemental Figure $7 \mathrm{H}$ for VGLUT3-constitutive-KO mice). During devaluation tests after RI training, as expected, control mice favored habitual behavior, as they exhibited comparable performance in valued and devalued conditions (Figure 3E and Supplemental Figure 7, I and J). In contrast, VGLUT3cKO mice and VGLUT3-constitutive-KO mice favored goal-directed behaviors, as they both performed less in the devalued condition (Figure 3E and Supplemental Figure 7, I and J). Male VAChTcKO mice nosepoked more than littermate controls during RI training (Supplemental Figure 7K). During devaluation tests after RI training, both control mice and VAChTcKO mice (males) exhibited habitual behavior by performing similarly in valued and devalued conditions (Supplemental Figure 7, L and $\mathrm{M})$. These results suggest that interfering with ChI cotransmission by silencing glutamate (VGLUT3cKO mice) causes mice to be less prone to develop habits, whereas shutting down ACh (VAChTcKO mice) increased the propensity of mice to habit formation. Thus, ACh and Glu released from ChIs have opposite roles in the modulation of goal-directed behaviors and habits.

Striatal cholinergic transmission regulates motivated behavior (32). To test whether increased habitual behavior demonstrated by VAChTcKO mice could be explained by changes in motivation, we compared male and female VAChTcKO mice 
A
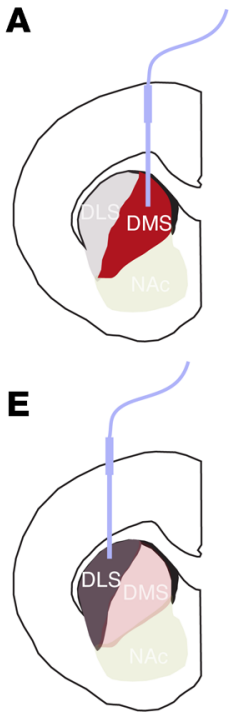

I

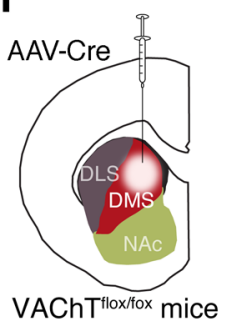

B

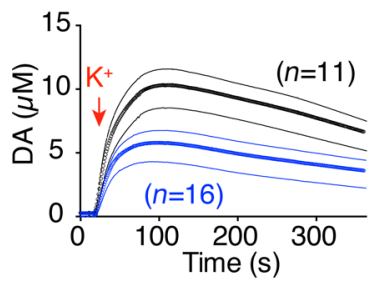

$\mathbf{F}$

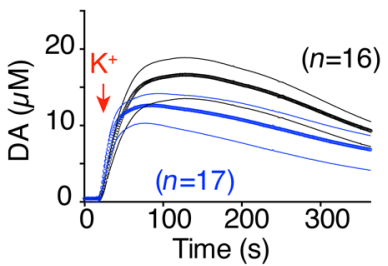

C

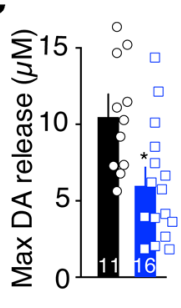

D

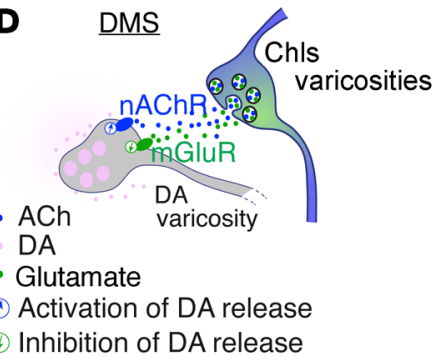

G

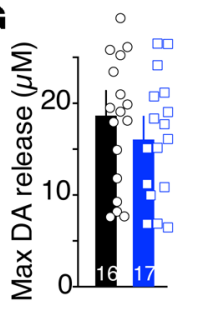

H

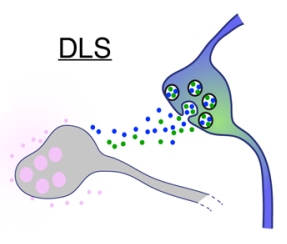

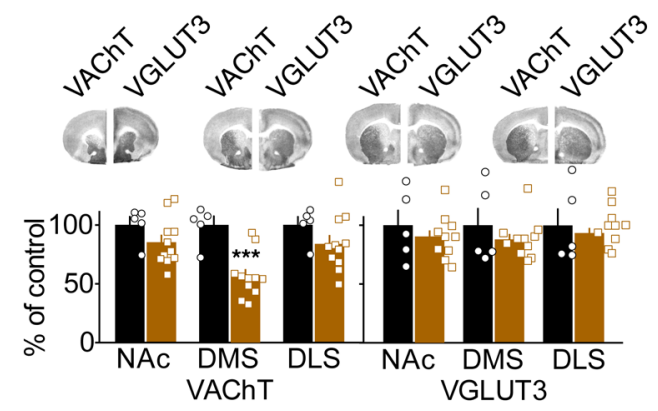
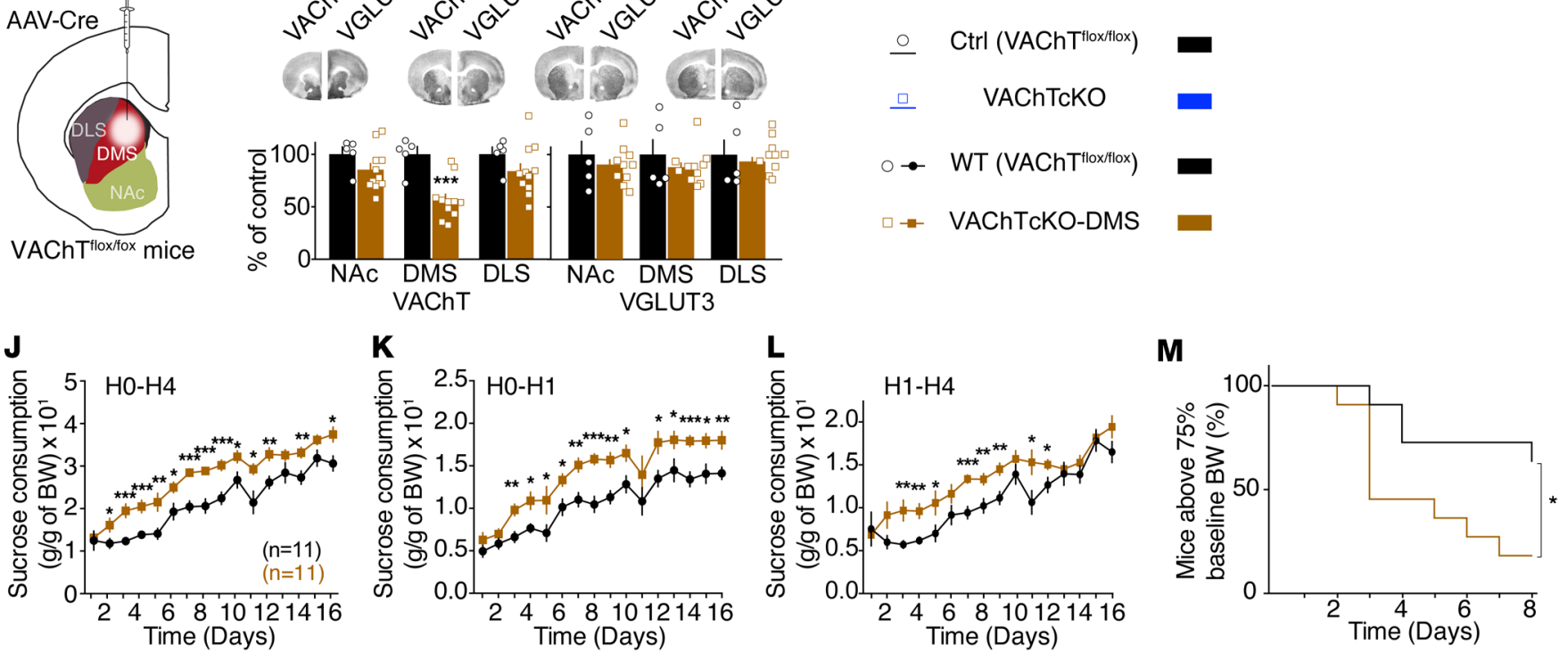

Figure 6. VAChT in the dorsomedial striatum regulates dopamine efflux and maladaptive eating. (A) Targeting of the dorsomedial subcompartment of the striatum (DMS) by voltammetric electrodes. NAc, nucleus accumbens. (B and C) DA release (B) and maximum level of DA release (C) in the DMS of VAChTcKO mice $(n=16)$ and controls $(n=11)$. (D) Putative model for dual regulation of striatal DA efflux by ACh/Clu released by Chls varicosities in the DMS. DA efflux is stimulated by ACh through nicotinic receptors ( $\mathrm{nAChRs)}$ ) and inhibited by glutamate through metabotropic glutamate receptors (mCluRs). (E) Targeting of the dorsolateral subcompartment of the striatum (DLS) by voltammetric electrodes. (F and $\mathbf{G})$ DA release (F) and maximum level of DA release (C) in the DLS of VAChTcKO mice $(n=16)$ and controls $(n=17)$. (H) The absence of regulation of DA release by ACh/Glu released by ChI varicosity in the DLS. (I) Quantification of VAChT (left) and VGLUT3 (right) protein expression following infusion of AAV-Cre or AAV-GFP viruses in the DMS of VAChT ${ }^{\text {fl/fl }}$ mice (VAChTCKO-DMS, $\left.n=11\right)$ or control mice ( $n=11$ ). (J-L) VAChTCKO-DMS mice and controls in the binge-like sucrose overconsumption model. (J) Daily sucrose consumption during the total duration of access (HOH4) for VAChTcKO-DMS mice and controls. (K) Similar to J but for HO-H1. (L) Similar to J but for H1-H4. (M) ABA model. Percentage of VAChTcKO-DMS mice and controls that reached a critical level of less than $75 \%$ baseline BW compared with controls during ABA. Two-way repeated-measures ANOVA (B, F, and J) and post hoc comparison with the method of contrasts; unpaired 2-tailed $t$ test (C, G, and I); Kaplan-Meier test (M) and post hoc comparison with log-rank Mantel-Cox and Gehan-Breslow-Wilcoxon tests. ${ }^{*} P<0.05,{ }^{* *} P<0.01,{ }^{* * *} P<0.001$.

in a progressive ratio (PR) test (Figure 3, $\mathrm{H}$ and $\mathrm{K}$ ). Cohorts of mice used in PR tests were the same as those previously used for FR1 training and devaluation tests (Supplemental Figure 8). VAChTcKO males demonstrated enhanced motivation compared with controls (Figure 3H), whereas VAChTcKO females and controls showed a similar breaking point in the PR test (Figure 3K). Thus, motivation may explain why males nosepoked more than controls during FR1 training. However, motivation was not a major determinant when VAChTcKO female mice performed in FR1. Furthermore, these behaviors were not due to increased locomotor activity, since VAChTcKO mice are not hyperactive in a novel or in a familiar environment (Supplemental Figure 7N). VGLUT3-deleted male mice showed no increased motivation in the PR test (Supplemental Figure 7O). Taken together, these find- 
A

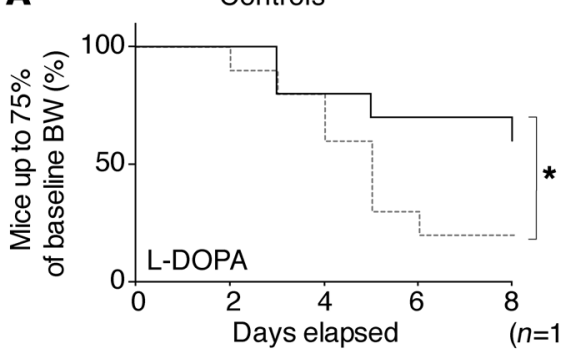

C

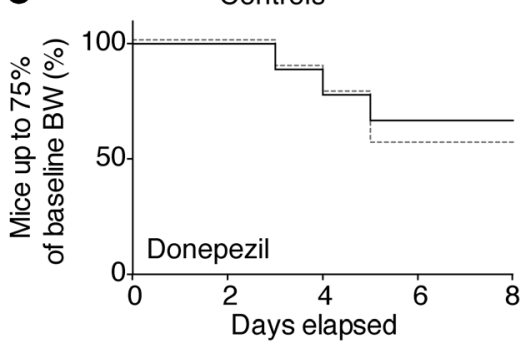

B

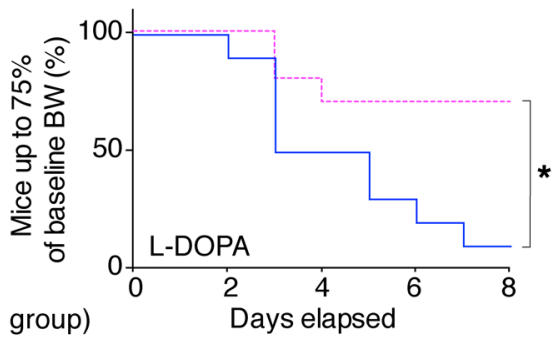

WT-NaCl (VAChTHoxillox) -
WT-L-DOPA (VAChT WT-donepezil (VAChT ${ }^{\text {Hoxthox}}$ ) -.-

D

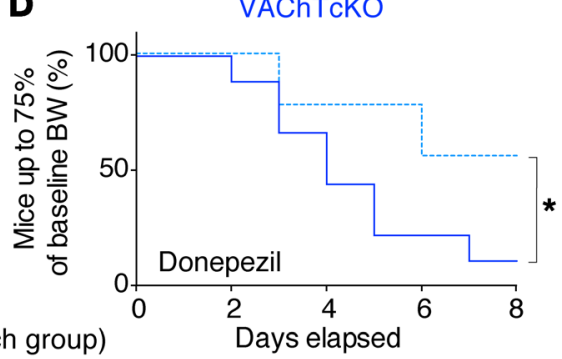

VAChTcKO-NaCl VAChTCKO-L-DOPA -.-

VAChTcKO-donepezil -----
E

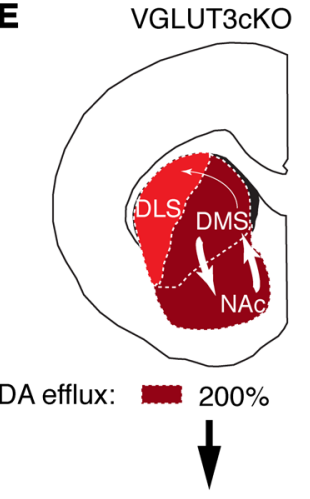

Goal-directed behaviors

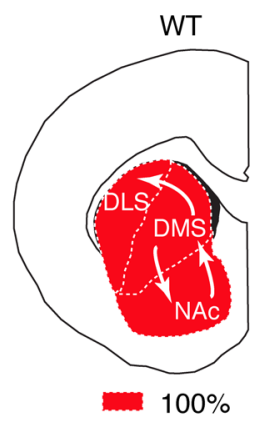

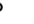

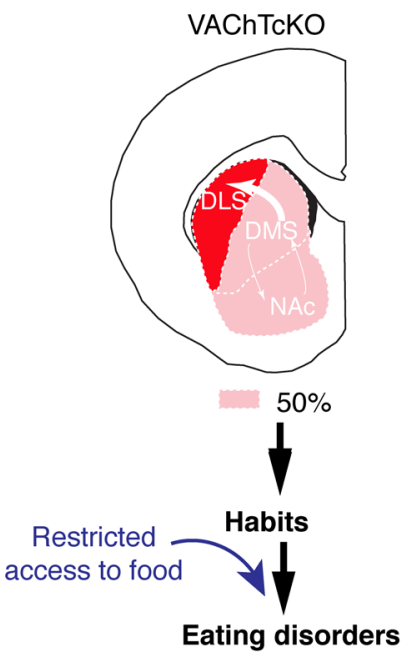

Figure 7. Pharmacological modulation of ACh or DA neurotransmission reverses the self-starvation phenotype observed in VAChTcKO mice. (A and B) Percentage of mice that reached critical weight threshold for control $(n=10)$ or VAChTcKO mice $(n=10)$ treated with saline or L-DOPA (15 mg/kg). A total of 9-10 were used in each group. (B and C) Chronic pharmacological treatment with donepezil $(0.3 \mathrm{mg} / \mathrm{kg})$. Percentage of mice that reached critical weight threshold for control $(n=10)$ or VAChTcKO mice $(n=10)$ treated with saline or donepezil. Note that donepezil was effective to reverse decreased food intake observed for VAChTcKO mice compared with saline-treated (NaCl-treated) VAChTcKO mice and had no effect on control mice. Kaplan-Meier test (A-D) and post hoc comparison with log-rank Mantel-Cox and Gehan-Breslow-Wilcoxon tests. ${ }^{*} P<0.05$. (E) Hypothetical model. Subcompartments of the striatum: (i) nucleus accumbens (NAc), reward-guided behaviors; (ii) dorsomedial striatum (DMS; or caudate in human), goal-directed behaviors; and (iii) dorsolateral striatum (DLS; or putamen in human), habits. Transitions from the NAc to the DMS and then to the DLS parallel the transition from voluntary reward-seeking behavior to compulsive behaviors. These transitions are finely tuned by dopamine (12). In VCLUT3cKO mice, DA effluxes are increased in the NAC/DMS but unchanged in the DLS. VCLUT3CKO mice are resistant to habit formation and remain in goal-directed behaviors. In contrast, in VAChTCKO mice, DA transmission is reduced by about 50\%-70\% in the NAc/DMS but remains unaltered in the DLS. Imbalanced DA levels in the NAc/DMS versus the DLS lead to rapid transitions from goal-directed behaviors to habits. In control conditions, VAChTcKO mice feed normally. Stressful conditions (e.g., restricted access to food) precipitate maladaptive eating behaviors in VAChTcKO mice.

ings suggest that VAChTcKO mice combine a lack of behavioral flexibility with an increased propensity to form habits, whereas VGLUT3cKO mice are resistant to habit formation.

VAChTcKO mice are more susceptible to dysfunctional eating. Deficits of behavioral flexibility and an increased tendency to develop habits are associated with pathological eating (Figure 1, G-I; and refs. 7, 33, 34). Thus, we tested whether VAChTcKO mice (with a behavioral flexibility deficit and more prone to habits) or VGLUT3cKO mice (more inclined to maintain goal-directed behaviors) were susceptible to maladaptive eating.

We first assessed binge-like sucrose overconsumption (35). Mice were food-restricted for 20 hours and then exposed simultaneously to food and to a highly concentrated sucrose solution for 4 hours each day, for 16 days (Figure 4A). This protocol was 
designed to mimic some human features of bulimia-like binge eating (35). VGLUT3cKO mice consumed sucrose, water, and food to a similar extent compared with littermate controls (Supplemental Figure 9, A-D). In contrast, during the 16 days of the test, both male and female VAChTcKO mice progressively exhibited a significant increase in sucrose consumption during the 4-hour session when compared with respective controls (Figure 4, B and E). The difference in sucrose consumption between VAChTcKO mice and controls was due to increased sucrose consumption in the first hour of access to sucrose ( $\mathrm{HO}-\mathrm{H} 1$; Figure 4, C and F). Sucrose intake between the second and the fourth hour was only minimally altered in VAChTcKO mice (H1-H4; Figure 4, D and $G)$. Water or food consumption during the first hour or during the total 4 hours of the test was similar between VAChTcKO mice and controls (Supplemental Figure 10, A-H). We also used saccharine, a non-caloric sweetener, in the binge-like model. VAChTcKO mice showed a small but significant tendency to binge more saccharine than controls (Supplemental Figure 10, I-L). These findings suggest that differences in homeostatic state do not contribute to sucrose overconsumption of VAChTcKO mice.

Eating high-caloric rewarding food without any physical hunger is an important feature of binge eating. Thus, we investigated whether palatability (hedonic effect) or homeostasis (hunger effect) contributed to the binge eating. The sucrose preference test was used to assess hedonia-driven motivation. Male VAChTcKO mice had a similar preference for the sucrose solution compared with control mice (Supplemental Figure 10N), indicating that the observed binge-like behavior was not caused by an increased sensitivity to the motivational properties of sucrose. We also examined the effect of reduced metabolic need (or hunger) on sucrose binge by measuring sucrose consumption after exposing animals to (a) an intraperitoneal injection of glucose solution 30 minutes before the session (Supplemental Figure 10, part O) or (b) ad libitum food for 1 hour ("chow preload"; Supplemental Figure 10P). In both conditions, VAChTcKO mice still binged significantly more sucrose than controls. Notably, VAChTcKO mice did not consume more food than controls during the chow preload period (Supplemental Figure 10Q). Furthermore, when appetitive food (highfat, high-sugar pellets) was used instead of regular food, mutant mice continued to preferentially binge more sucrose solution than control mice (Supplemental Figure 10R), although they consumed the same amount of the alternative reward (Supplemental Figure 10S). These findings suggest that metabolic differences were not involved in the sucrose overconsumption exhibited by VAChTcKO mice during the first hour $(\mathrm{HO}-\mathrm{H} 1)$ of the test. Thus, VAChTcKO mice, which showed accelerated habit formation, exhibited a higher tendency to develop a binge-like sucrose overconsumption irrespectively of hunger or metabolic needs.

We next assessed VAChT- and VGLUT3-mutant mice in the activity-based anorexia (ABA) model, a well-validated rodent model of maladaptive eating endophenotype related to anorexia nervosa (Figure $4 \mathrm{H}$ ) (36). Mice were habituated for 7 days to individual cages with free access to a running wheel. During this baseline period, mutant mouse lines and controls showed no differences of food intake or body weight (Supplemental Figure 11, A-F). A progressive time-restricted access to food was then introduced during the following 8 days, pushing mice to paradoxically combine overexercise and voluntary food restriction. During the ABA food restriction period, VGLUT3cKO mice and littermate controls showed similar food intake and reached $75 \%$ of their baseline body weight at comparable rates (Supplemental Figure $11, \mathrm{G}$ and $\mathrm{H})$. In contrast, both male and female VAChTcKO mice demonstrated significant alteration of food intake relative to control littermates (Supplemental Figure 11I). In addition, compared with controls, more male and female VAChTcKO mice reached a threshold of less than $75 \%$ of their baseline body weight (i.e., a critical weight loss as observed in patients with anorexia nervosa; ref. 1) (Figure 4, I and J). Together, these results suggest that, when VAChTcKO mice have limited access to food, they are more likely to develop maladaptive/compulsive eating behaviors.

Increased propensity to habit formation drives maladaptive eating in VAChTcKO mice. VAChTcKO mice demonstrated a propensity to develop habits as well as vulnerability to maladaptive eating. Since we used the same cohort of VAChTcKO mice and littermate controls to perform several behavioral tests (Supplemental Figure 8), we next assessed the link between habits, motivation, sex, and maladaptive eating behavior. We first performed Pearson correlations (Figure 5, A-D; and Supplemental Figure 12).

We then used correlational data to build a structural equation model (Figure 5, E and F) using latent variable analysis to test the effects of habits and motivation on anorexia- and bulimia-like behaviors, simultaneously. This analysis revealed that excessive habitual behavior predicts vulnerability of male and female VAChTcKO mice to maladaptive eating behavior (Figure 5, E and F).

Using logistic regression and receiver operating characteristic curves (ROCs), we then tested the diagnostic power of valuation/ devaluation scores to identify mice's vulnerability to binge-like behavior (Figure 5G) and anorexia-like behavior (Figure $5 \mathrm{H}$ ). Valuation scores and sex were significant predictors of binge-like behaviors with a diagnostic power of $94.4 \%$ (area under the ROC curve), and devaluation scores were significant predictors of vulnerability to anorexia-like behavior with $92.1 \%$ diagnostic power (Figure $5 \mathrm{H}$ ). Thus, our results suggest that cholinergic dysfunction due to VAChT deletion from ChIs drives habitual behaviors and vulnerability to maladaptive eating behaviors (binge- and anorexia-like) under specific environmental conditions. Our data also suggest that goal-directed and habitual behavior scores could be used to predict vulnerability to the development of binge-like and anorexia-like behaviors in mutant mice.

$D A$ efflux is differentially affected across the dorsal striatum of $V A C h T c K O$ mice. Local DA neurotransmission plays a central role in the striatum and is thought to be pivotal in regulating reward, the balance between goal-directed behaviors and habits, and cognitive flexibility $(12,37)$. It is well established that cholinergic signaling regulates striatal DA neurotransmission $(13,14)$. In the nucleus accumbens, ACh and Glu released by ChIs differentially regulate DA efflux (13). To determine whether disruption of DA neurotransmission in VAChTcKO mice could be related to the maladaptive eating phenotypes, we measured DA efflux in the dorsal striatum. Microdialysis experiments showed that elimination of VAChT from ChIs decreased extracellular DA accumulation in the dorsal striatum by $50 \%$ (Supplemental Figure 13A). We then used in vivo voltammetry to measure DA efflux in the dorsomedial striatum and in the dorsolateral striatum of VAChTcKO mice, VGLUT3cKO 
mice, and littermate controls (Figure 6, A-H; and Supplemental Figure 13, B-Q). Following $\mathrm{KCl}$-induced depolarization, DA release was decreased in the dorsomedial striatum of VAChTcKO mice $(-40 \%)$ (Figure 6, B and C; and Supplemental Figure 13P) compared with control mice. Conversely, it was increased in the dorsomedial striatum of VGLUT3cKO mice (+40\%) (Supplemental Figure 13, B, C, and P, for VGLUT3cKO, and Supplemental Figure $13, \mathrm{~F}-\mathrm{H}$, for VGLUT3-constitutive-KO mice). In the dorsolateral striatum, DA efflux was not modified after deletion of either VAChT or VGLUT3 (Figure 6, F and G; and Supplemental Figure 13, D, E, and Q, for VAChTcKO and VGLUT3cKO, and Supplemental Figure 13, I-K, for VGLUT3-KO mice). Therefore, disturbing ACh secretion from ChIs markedly affects DA release in the dorsomedial striatum without altering its levels in the dorsolateral striatum. Opposite changes are observed in the absence of glutamate secretion from ChIs. Overall, altering ACh/Glu cotransmission in ChIs creates an imbalance in DA release between subcompartments of the dorsal striatum.

Deletion of VAChT in the dorsomedial striatum of male mice is sufficient to induce maladaptive eating behavior. DA voltammetry experiments suggest that reduced levels of VAChT affect DA efflux in the dorsomedial striatum, but not in the dorsolateral striatum. Therefore, we tested whether altered VAChT levels in the dorsomedial striatum could be involved in maladaptive eating. To address this question, we specifically deleted VAChT from the dorsomedial striatum of adult male mice by infusing AAV-Cre virus in this striatal subcompartment (VAChTcKO-DMS; Figure 6I) and tested them for maladaptive eating. Strikingly, in the binge-like sucrose overconsumption model, decreased expression of VAChT restricted to the dorsomedial striatum was sufficient to reproduce the increased sucrose intake (Figure 6, J-L) observed in VAChTcKO mice (Figure 4, B-G). Likewise, throughout the 16 days of binge-like testing, neither food nor water intake was modified in VAChTcKO-DMS mice relative to controls (Supplemental Figure 14, A and B). In addition, VAChTcKO-DMS mice had a similar preference for the sucrose solution compared with controls (Supplemental Figure 14C). Furthermore, as reported above for VAChTcKO mice, VAChTcKO-DMS mice that were satiated by pre-exposure to chow for 1 hour still binged more sucrose than controls (Supplemental Figure 14D). Importantly, there was no difference in food intake during this chow preload period (Supplemental Figure 14E). Therefore, increased binge activity in VAChTcKO-DMS mice was not related to hunger, but most likely was related to increased habitual behavior.

In the ABA model, more mice reached critical levels of weight loss following the deletion of VAChT in the dorsomedial striatum compared with controls (Figure 6M). Interestingly, we observed no correlation between the extent of VAChT deletion in the dorsomedial striatum and sucrose binge-like overconsumption or self-starvation phenotypes (Supplemental Figure 14, F and G). These correlative analyses suggest that even a partial deletion of VAChT in the dorsomedial striatum could be sufficient to increase vulnerability to maladaptive eating. Importantly, when we analyzed between-subject differences in sucrose binge-like overconsumption and ABA models, no correlation was found for control mice (Supplemental Figure 14H). Conversely, Pearson correlation analyses yielded a robust negative correlation between the quantity of sucrose consumed during the binge-like model and food intake in the ABA model for VAChTcKO-DMS mice (Supplemental Figure 14I). Hence, VAChTcKO-DMS mice that were more vulnerable to bulimia-like phenotype were also more susceptible to anorexia-like behavior 10 days later. These data show that a limited disruption of cholinergic activity in the dorsomedial striatum of adult mice is sufficient to increase vulnerability to maladaptive eating.

Pharmacological modulation of ACh or DA neurotransmission reverses the $A B A$ phenotype of $V A C h T C K O$ mice. To test whether the reduced DA release observed in VAChT mutants is related to maladaptive eating behaviors, we treated male VAChTcKO mice and controls with L-DOPA $(15 \mathrm{mg} / \mathrm{kg})$ during the ABA test. We chose the ABA model because of the robust phenotype of VAChTcKO mice in this test. During the baseline phase, the presence of the wheel did not alter food intake or body weight of VAChTcKO mice and controls (Supplemental Figure 15, A-D). During the restriction phase, L-DOPA exacerbated the anorexia-like phenotype of control mice as shown by survival curves (Figure 7A and Supplemental Figure $15 \mathrm{E}$ ) and as previously reported (38). In contrast, the percentage of VAChTcKO mice reaching a critical weight loss was greatly reduced by L-DOPA pretreatment (Figure 7B and Supplemental Figure 15E). Therefore, increasing DA transmission with L-DOPA alleviated the self-starvation phenotype of VAChTcKO mice, although an opposite effect was observed in littermate controls. These data indicate that increasing DA efflux can rescue maladaptive eating deficits in VAChT-deficient mice, suggesting that decreased DA is causally linked to the behavioral deficits in these mutants.

Donepezil is an acetylcholinesterase (AChE) inhibitor that reverses deficits of VAChT-mutant mice (39). To confirm that the phenotypes observed in VAChT-deficient mice could be due to reduced cholinergic signaling, we tested whether donepezil was able to reverse self-starvation phenotypes in the ABA model. Chronic treatment with donepezil $(0.3 \mathrm{mg} / \mathrm{kg})$ had no effect on the percentage of control mice reaching a critical threshold of less than $75 \%$ of their baseline body weight (Figure 7C and Supplemental Figure $15 \mathrm{~F}$ ). In contrast, donepezil significantly decreased the percentage of VAChTcKO mice reaching the $75 \%$ threshold of baseline body weight (Figure 7D and Supplemental Figure 15F). Thereby, increasing cholinergic signaling by AChE inhibition was fully effective to treat the anorexia-like phenotype in VAChTcKO mice.

Figure $7 \mathrm{E}$ summarizes our findings in animal models. In this hypothetical model, we suggest that an unbalanced DA transmission across striatal subcompartments alters the transition from goal-directed behaviors to habits and drives the vulnerability of VAChTcKO mice to the development of environment-dependent maladaptive eating.

\section{Discussion}

ChIs have the remarkable ability to corelease ACh and glutamate $(17,40)$. In this study, we show a striking dissociation of neurochemical and behavioral actions modulated by these 2 neurotransmitters in the dorsal striatum. When glutamate transmission from ChIs is silenced by deletion of VGLUT3, DA efflux increases in the dorsomedial striatum but not in the dorsolateral striatum. In contrast, decreased release of ACh due to VAChT deletion results in decreased DA efflux in the dorsomedial striatum, but not in the dorsolateral striatum (as modeled in Figure 7E). Reducing gluta- 
mate transmission from ChIs (VGLUT3cKO mice) favors goal-directed behaviors, and these mice are not more vulnerable to maladaptive eating than littermate controls. In contrast, VAChTcKO mice present a loss of behavioral flexibility and facilitated habit formation that predict their increased risk of developing maladaptive eating behaviors. Importantly, L-DOPA treatment reversed the self-starvation phenotype in VAChT-deficient mice, supporting the interpretation that reduced DA efflux may play a pivotal role in the maladaptive eating observed in these mice. We surmise that, in addition to signaling mechanisms elicited by ACh, favoring DA efflux in the dorsolateral striatum versus the dorsomedial striatum/nucleus accumbens could directly lead to excessive habits and bulimia-like or anorexia-like behaviors. Future experiments will help to establish whether specifically decreasing dopamine efflux in the dorsomedial striatum/nucleus accumbens or increasing it in the dorsolateral striatum (as described in Figure 7E) is sufficient to accelerate habit formation and maladaptive eating.

We previously found that silencing ACh or Glu released by ChIs differently affected DA release in the nucleus accumbens and reward-based behaviors $(13,18)$. Glutamate released from ChIs inhibits DA transmission in the nucleus accumbens by activation of a metabotropic glutamate receptor (mGluR) (13). Glutamate released by ChIs could operate on inhibitory group I mGluR receptors present on DA striatal fibers (41). In addition, it is well established that a combination of striatal nicotinic and muscarinic receptors accounts for the local regulation of DA signaling (13, $14,41,42)$. Our findings, combined with data from the literature, support a framework in which ACh/Glu cotransmission from ChIs exerts a dual effect on DA release in both the nucleus accumbens and the dorsomedial striatum, but not in the dorsolateral striatum (Figure 7E). This difference between the nucleus accumbens/dorsomedial striatum and the dorsolateral striatum is likely due to the presence of a distinct combination of mGluRs, nicotinic AChRs, and muscarinic AChRs present in DA fibers in various striatal subcompartments (41). ACh and Glu from ChIs modulate the balance of DA transmission between striatal compartments and, by doing so, play a pivotal role in the regulation of the transition from goal-directed behaviors to habits. An imbalance of cotransmission favoring ACh release correlates with increased goal-directed behaviors, whereas an imbalance favoring glutamate release correlates with habitual behaviors.

Targeted deletion of VAChT in the dorsal striatum of adult mice using viral vectors recapitulates the loss of behavioral flexibility as well as sucrose overconsumption and self-starvation observed in VAChTcKO mice. These results rule out the involvement of developmental compensatory mechanisms in endophenotypes herein uncovered. In addition, specific deletion of VAChT in the dorsomedial striatum suggests that this striatal area is sufficient to drive maladaptive eating behaviors. However, these findings do not rule out the possibility that other brain areas could also fulfill similar functions. The striatum is part of the complex cortico-striato-thalamo-cortical loop, and the prefrontal cortex is a pivotal regulator of this neuronal network. Several reports from the literature suggest that the prefrontal cortex, and in particular the orbitofrontal cortex, are critical for the regulation of reward, compulsivity, and eating disorders $(43,44)$. In addition, several brain areas such as other structures from the cortico-basalo-cor- tical loop, the thalamus, the hippocampus, or the amygdala play an influential role in goal-directed or habitual actions (10, 22, 45). Our results combined with data from the literature suggest that the dorsal striatum could act as a final endpoint effector of a pathway driven by the prefrontal cortex.

In parallel, we found that the maladaptive eating of VAChTcKO mice cannot be attributed to locomotor hyperactivity, anhedonia, impulsivity, motivation, or metabolic need. We also observed that mice with VAChT deletion in the dorsomedial striatum that binged more sucrose also showed the lowest food intake in the ABA model a few days later. Altogether, these findings further suggest that maladaptive eating behaviors observed in VAChTcKO mice correlates with decreased $\mathrm{ACh}$ transmission in the dorsal striatum and increased habit formation. Interestingly, in a familiar environment (such as a regular home cage), feeding behavior of VAChTcKO mice and controls is similar (the present study and ref. 46). Abnormal eating behaviors of mice with dysfunctional striatal cholinergic transmission occur only in conditions such as restricted access to food. These findings illustrate how genetic and environmental factors may interact to drive abnormal eating behaviors.

There have been conflicting data over the involvement of excessive and maladaptive habits in eating disorders $(25,47)$. Using a self-reported habit index, Davis et al. recently found that the chronicity and severity of anorexia nervosa were related to the strength of habits (48). Using a neurocognitive task previously designed to assess habit formation in humans $(24,25)$, we showed that a subgroup of patients with restrictive anorexia nervosa present an increased tendency to habits. Our present findings are well in line with a recent report (44), which combined an instrumental motivation task and functional MRI in acutely underweight anorexic patients. This study unraveled the existence of a subgroup of anorexic patients favoring goal-directed actions (gAn) and a second subgroup that was more habit driven (hAn) (44).

In our study, we observed that enhanced habit formation was strongly correlated with decreased cognitive flexibility in restrictive anorexia nervosa patients but not binge-purge anorexia nervosa patients. The human test used herein is based on a go/no-go inhibitory task, whereas the mouse task relies on a pre-session outcome devaluation of the reinforcer. However, despite these differences, these tests support the idea that excessive habits can push both humans and mice to use automatic behaviors and to become less sensitive to the value of the outcome. In humans, these cognitive investigations suggest that the propensity to quickly develop habitual behavior combined with a lack of cognitive flexibility could serve as behavioral markers for a subgroup of restrictive anorexia patients.

Clinically it is well established that eating disorders, especially anorexia nervosa and bulimia nervosa, are more frequent among females than males (1). Unlike humans, VAChTcKO mice demonstrated only minor differences between males and females in maladaptive eating behavior. Nonetheless, our findings show that these mice recapitulate some of the major endophenotypes of eating disorders in humans. Another discrepancy between patients and our animal model is that habit formation in VAChTcKO mice predicted vulnerability to both anorexia-like and bulimia-like behaviors. Our data showed facilitation of habits in a subgroup of restrictive anorexia nervosa patients, whereas binge-purge anorexia nervosa patients demonstrated no change of habit for- 
mation. This might reflect an intrinsic difference between human patients and the animal model. However, it could also be related to the fact that we assessed habits in a small cohort of patients with bulimia nervosa. The neurocognitive test was conducted with a large majority of anorexic patients (binge-purge anorexia or restrictive anorexia) and included only 7 patients with bulimia nervosa in the ED-BP group (Figure 1 and Supplemental Table 1). To clarify whether excessive habits can also drive the symptoms of bulimia nervosa, further studies with a large cohort of subjects will be necessary.

Several reports previously proposed compulsivity as a transdiagnostic marker overlapping different eating disorders $(5,7$, $25,48)$. Our findings taken together with observations by Davis et al. $(48,49)$ suggest that increased habit formation could be a transdiagnostic marker for subgroups of anorexia nervosa as well as bulimia nervosa patients. Interestingly, excessive goal-directed behavior has also been proposed as a transdiagnostic marker for subgroups of eating disorders patients (44). On that note, it is tempting to hypothesize that in our study (Figure 1E), patients who do less than 10\%-20\% mistakes in devalued conditions could represent goal-driven patients. If this hypothesis is correct, evaluation of habits versus goal-directed behaviors could be an innovative way to more specifically stratify patients with eating disorders. Precisely defining these subgroups could be key to better understand and treat these pathologies.

The present study focuses on one endophenotype of anorexia, i.e., excessive habit formation, and on the network involving the caudate-putamen. However, it should be kept in mind that anorexia nervosa is a complex pathology and several important dimensions are not easily modeled in animal studies. This is the case, for example, with obsessional preoccupation with body image, severe body image distortions, or lack of insight about being ill. These dimensions have been proposed to involve other transmitters than $\mathrm{ACh}$ and DA and other brain areas than the cortico-basalo-cortical loop (45). These additional dimensions and brain areas will have to be investigated with appropriate animal models in order to develop effective treatment of anorexia.

Deficits of DA or ACh transmission can be pharmacologically alleviated with L-DOPA or donepezil, respectively. Indeed, we found that L-DOPA was efficient in reducing anorexia-like symptoms in VAChTcKO mice. This observation supports the idea that a reduced DA efflux in the dorsomedial striatum/nucleus accumbens could play a causative role in establishing maladaptive eating behaviors in VAChTcKO mice (Figure 7E). However, L-DOPA markedly increased anorexia-like behaviors in control mice. In humans, L-DOPA induces a range of effects: it can lead to anorexic symptoms (50), cause antianorexic effects (51), or have no effect (52). An inverted $U$ shape of dopamine effects has often been reported (53). Therefore, paradoxical effects of L-DOPA in controls versus VAChTcKO mice could be related to the notion that too much or too little dopamine are both detrimental. Therefore, DA-targeting drugs or L-DOPA should be used with care for the treatment of eating disorders.

In contrast, we observed that the AChE inhibitor donepezil prevented the self-starvation phenotype of VAChTcKO mice without altering eating behaviors of control mice. This finding supports the idea that abnormal striatal cholinergic signaling correlates with increased vulnerability to maladaptive eating in VAChTcKO mice. Donepezil was previously proposed to treat anorexia (54); however, the rationale underlying this suggestion emerged from cognitive parallels drawn between dementia and anorexia, which are quite different from the model herein proposed (Figure 7E). AChE inhibitors have also been proposed to rectify eating compulsions in Alzheimer's disease $(55,56)$. However, the efficacy of procholinergic drugs in the treatment of anorexia is still controversial $(57,58)$. Interestingly, donepezil has been demonstrated to be a well-tolerated and efficient add-on drug for treatment-resistant obsessive-compulsive disorder and pathological repetitive behaviors (tics) $(59,60)$. Our present findings suggest that it might be relevant to test AChE inhibitors in eating disorder-affected individuals who present increased habitual behaviors. However, despite a relatively favorable profile of side effects, AChE inhibitors can cause nausea and diarrhea and should therefore be used with caution to treat this population of patients. In particular, low doses should be favored in potential future clinical trials.

It could be worthwhile to assess whether subgroups of patients with eating disorders exhibit genetic alterations of VAChT or other cholinergic striatal markers before treating them with donepezil. Notably, VAChT probes used in positron emission tomography have been described (61). Subjects with altered expression of cholinergic markers and a higher propensity to habit formation would then be candidates to undergo cholinergic-enhancing therapies. It is important to note that altering the function of ChIs is not the sole way to modify the balance between reward-guided goal-directed behavior and habit formation. This could also be achieved, for example, by modification of the activity of the orbitofrontal cortex $(43,62)$ (Figure 7 ) or by inhibition of cholinergic neurons from the midbrain projecting to the striatum (63).

In summary, our work indicates that enhanced habit formation can promote vulnerability to maladaptive eating in mice as well as in subgroups of human patients. Our data open avenues to the identification of a specific subgroup of patients suffering from restrictive anorexia nervosa or bulimia nervosa and suggest potential innovative targeted therapies for the treatment of these patients.

\section{Methods}

Human subjects. Description of patients and healthy controls is provided in Supplemental Methods.

The slip-of-action neurocognitive test. To evaluate the balance between goal-directed behaviors and habits in humans, we used a computer-based neurocognitive task as previously reported by (24, 25). The test is described in Supplemental Methods.

Animals. Housing and breeding of mouse colonies were performed as previously reported (13) and as described in Supplemental Methods.

Histology and cellular/molecular assays. Immunoautoradiography, in situ hybridization, Western blots, immunofluorescence, and quantitative PCR experiments were performed as previously reported (13, 64) and as described in Supplemental Methods. See complete unedited blots in the supplemental material.

Behavioral investigations. Touchscreen behavioral tasks, operant sucrose self-administration, sucrose binge-like overconsumption, sucrose preference, and ABA model were performed as previously reported $(31,35,36,65)$ and as described in Supplemental Methods. 
Stereotaxic surgery and intracerebral virus infusions. Details regarding virus injections and histological evaluations of VAChT deletion for touchscreen experiments and for eating disorder models are provided in Supplemental Methods.

In vivo microdialysis. Details regarding in vivo microdialysis experiments are described in Supplemental Methods.

In vivo voltammetry. In vivo voltammetry was performed as previously reported (13) and as briefly described in Supplemental Methods.

Data and materials availability. All relevant data and analyses are within the paper and its supplemental material online. Raw data are available upon request. All unique biological materials used (knockout mice or antiserums) are readily available upon request.

Statistics. Statistical analyses are shown in Supplemental Tables 3-21. Details regarding statistical analysis and mathematical modeling are provided in Supplemental Methods.

Study approval. Clinical studies were approved by the local ethics committee ("Comité de Protection des Personnes," CPP: AM714913321). A signed informed consent document was obtained from each participant before enrollment.

Animal care, animal handling, and all experiments were performed according to the guidelines of the Canadian Council on Animal Care (http://ccac.ca/en/standards/guidelines), approved by the Facility Animal Care Committee of the Douglas Research Center (protocols 2008-5643 and 2014-7479) or by the University of Western Ontario (protocols 2016-103 and 2016-104), and performed in accordance with the NIH guidelines for the care and use of animals and an approved animal protocol from the Duke University IACUC.

\section{Author contributions}

$\mathrm{MF}$ and $\mathrm{HJ}$ performed the behavioral experiments with the help of OK, XQM, CA, and SR. MF and HJ performed the anatomical experiments with the help of EV. MF performed in vivo DA voltammetry with the help of LM and AG. JIK programmed the computerized neuropsychological test. DJ performed the neuropsychological test in humans with the help of PD and under the supervision of PG. JPRJ performed in vivo microdialysis under the supervision of MGC. PRN, LR, JYN, TAP, and MMH performed statistical analysis and mathematical models. MF, MAMP, VFP, and SEM designed the study and wrote the manuscript with the help of PRN, HJ, JG, DG, MGC, MMC, and MPB. All authors were involved in revising the manuscript for intellectual content. All authors read and approved the final manuscript. MF and $\mathrm{HJ}$ are co-first authors. MF is listed first because he contributed more to the conception of the project and the writing of the manuscript.

\section{Acknowledgments}

The authors thank Fabio Marti, Timothy Bussey, Sebastien Carnicella, Jens-Jakob Karlsson, Julia Pedersen, Apolline Alfsen, Jue Fan, Matthew Cowan, Yeqing Geng, and Yulia Worbe for their help. MF and SEM are very grateful to Martin Hirsch and Catherine Dassault for their support. OK received an Ontario Graduate Student doctoral award. This research was supported by funds from the Brain Canada Multi-Investigator Research Initiative (to SEM, VFP, and MAMP), Fond de Recherche Santé Québec (FRQS 30582 to SEM), Natural Sciences and Engineering Research Council Discovery Grants (RGPIN/386431-2012 and RGPIN/04682-2017 to SEM), Fondation de France (to SEM), ERA-NET NEURON Joint Transnational Call for "European Research Projects on Mental Disorders" and research projects on synaptic dysfunction in disorders of the central nervous system (JTC 2013 and 2017 to SEM), the Djavad Mowafaghian Foundation (to SEM), Agence Nationale de la Recherche (ANR-13-SAMA-0005-01 to SEM), CNRS (to SEM), INSERM (to SEM), and Université Pierre et Marie Curie (to SEM). MAMP's and VFP's contributions to this work received support from the Canadian Institutes of Health Research (MOP 126000, MOP 136930, MOP 89919, PJT 162431, PJT 159781), the National Science and Engineering Research Council of Canada (402524-2013 RGPIN), the Weston Brain Institute (for development of touchscreen experiments), and a BrainsCAN/Canada First Research Excellence Fund Accelerator award. MAMP is a Tier I Canada Research Chair in Neurochemistry of Dementia. Parts of this work were also supported by NIH grants 5R37-MH073853 and 5R01-MH079201 (to MGC).

Address correspondence to: Salah El Mestikawy, Douglas Mental Health University Institute, Department of Psychiatry, McGill University - Pavillon Perry E-3213, 6875 Boulevard Lasalle, Montréal, Quebec H4H 1R3, Canada. Phone: 514.761.6131 ext. 6151; Email: salah.elmestikawy@mcgill.ca. Or to: Vania F. Prado or Marco A.M. Prado, Robarts Research Institute, Translational Neuroscience Group, Department of Anatomy and Cell Biology and Department of Physiology and Pharmacology, The University of Western Ontario, 1151 Richmond Street N, London, Ontario N6A 5B7, Canada. Phone: 519.931.5777 ext. 24889; Email: vprado@robarts.ca (VFP). Phone: 519.931.5777 ext. 24888; Email: mprado@robarts.ca (MAMP).
1. Fairburn CG, Harrison PJ. Eating disorders. Lancet. 2003;361(9355):407-416.

2. Arcelus J, Mitchell AJ, Wales J, Nielsen S. Mortality rates in patients with anorexia nervosa and other eating disorders. A meta-analysis of 36 studies. Arch Gen Psychiatry. 2011;68(7):724-731.

3. American Psychiatric Association. Diagnostic and Statistical Manual of Mental Disorders. 8th ed. American Psychiatric Association; 2013.

4. Trace SE, Baker JH, Peñas-Lledó E, Bulik CM. The genetics of eating disorders. Annu Rev Clin Psychol. 2013;9:589-620.

5. Banca P, Harrison NA, Voon V. Compulsivity across the pathological misuse of drug and nondrug rewards. Front Behav Neurosci. 2016;10:154

6. Friederich HC, Herzog W. Cognitive-behavioral flexibility in anorexia nervosa. Curr Top Behav Neurosci. 2011;6:111-123.

7. Godier LR, Park RJ. Compulsivity in anorexia nervosa: a transdiagnostic concept. Front Psychol. 2014;5:778.

8. Voon V, et al. Disorders of compulsivity: a common bias towards learning habits. Mol Psychiatry. 2015;20(3):345-352.

9. Steinglass JE, et al. Targeting habits in anorexia nervosa: a proof-of-concept randomized trial. Psychol Med. 2018;48(15):2584-2591.

10. Balleine BW, O'Doherty JP. Human and rodent homologies in action control: corticostriatal determinants of goal-directed and habitual action. Neuropsychopharmacology. 2010;35(1):48-69.

11. Belin-Rauscent A, Everitt BJ, Belin D. Intra- striatal shifts mediate the transition from drug-seeking actions to habits. Biol Psychiatry. 2012;72(5):343-345.

12. Wickens JR, Horvitz JC, Costa RM, Killcross S. Dopaminergic mechanisms in actions and habits. J Neurosci. 2007;27(31):8181-8183.

13. Sakae DY, et al. The absence of VGLUT3 predisposes to cocaine abuse by increasing dopamine and glutamate signaling in the nucleus accumbens. $\mathrm{Mol}$ Psychiatry. 2015;20(11):1448-1459.

14. Threlfell S, Lalic T, Platt NJ, Jennings KA, Deisseroth K, Cragg SJ. Striatal dopamine release is triggered by synchronized activity in cholinergic interneurons. Neuron. 2012;75(1):58-64.

15. Gras C, et al. A third vesicular glutamate transporter expressed by cholinergic and serotoniner- 
gic neurons. J Neurosci. 2002;22(13):5442-5451.

16. El Mestikawy S, Wallén-Mackenzie A, Fortin GM, Descarries L, Trudeau LE. From glutamate co-release to vesicular synergy: vesicular glutamate transporters. Nat Rev Neurosci. 2011;12(4):204-216.

17. Gras C, et al. The vesicular glutamate transporter VGLUT3 synergizes striatal acetylcholine tone. Nat Neurosci. 2008;11(3):292-300.

18. Guzman MS, et al. Elimination of the vesicular acetylcholine transporter in the striatum reveals regulation of behaviour by cholinergic-glutamatergic co-transmission. PLoS Biol. 2011;9(11):e1001194.

19. Aoki S, Liu AW, Zucca A, Zucca S, Wickens JR. Role of striatal cholinergic interneurons in set-shifting in the rat. JNeurosci. 2015;35(25):9424-9431.

20. Martos YV, Braz BY, Beccaria JP, Murer MG, Belforte JE. Compulsive social behavior emerges after selective ablation of striatal cholinergic interneurons. J Neurosci. 2017;37(11):2849-2858.

21. Prado VF, Janickova H, Al-Onaizi MA, Prado MA. Cholinergic circuits in cognitive flexibility. $\mathrm{Neu}$ roscience. 2017;345:130-141.

22. Bradfield LA, Bertran-Gonzalez J, Chieng B, Balleine BW. The thalamostriatal pathway and cholinergic control of goal-directed action: interlacing new with existing learning in the striatum. Neuron. 2013;79(1):153-166.

23. de Wit S, Watson P, Harsay HA, Cohen MX, van de Vijver I, Ridderinkhof KR. Corticostriatal connectivity underlies individual differences in the balance between habitual and goal-directed action control. JNeurosci. 2012;32(35):12066-12075.

24. Worbe Y, Savulich G, de Wit S, Fernandez-Egea E, Robbins TW. Tryptophan depletion promotes habitual over goal-directed control of appetitive responding in humans. Int J Neuropsychopharmacol. 2015;18(10):pyv013.

25. Godier LR, et al. An investigation of habit learning in anorexia nervosa. Psychiatry Res. 2016;244:214-222.

26. Gremel CM, Costa RM. Orbitofrontal and striatal circuits dynamically encode the shift between goal-directed and habitual actions. Nat Commun. 2013;4:2264.

27. Matamales M, Skrbis Z, Hatch RJ, Balleine BW, Götz J, Bertran-Gonzalez J. Aging-related dysfunction of striatal cholinergic interneurons produces conflict in action selection. Neuron. 2016;90(2):362-373.

28. Aoki S, Liu AW, Akamine Y, Zucca A, Zucca S, Wickens JR. Cholinergic interneurons in the rat striatum modulate substitution of habits. Eur J Neurosci. 2018;47(10):1194-1205

29. Guevara BH, Talmaciu RK, Hoffmann IS, Cubeddu LX. Comparative dopamine-acetylcholine interactions in the ventral and dorsal striatum of rabbit and rat brain. Brain Res. 1996;733(1):105-107.

30. Jongen-Rêlo AL, Docter GJ, Jonker AJ, Voorn P. Differential localization of mRNAs encoding dopamine D1 or D2 receptors in cholinergic neurons in the core and shell of the rat nucleus accumbens. Brain Res Mol Brain Res. 1995;28(1):169-174.

31. Rossi MA, Yin HH. Methods for studying habitual behavior in mice. Curr Protoc Neurosci. 2012; Chapter 8:Unit 8.29.

32. Collins AL, Aitken TJ, Greenfield VY, Ostlund SB,
Wassum KM. Nucleus accumbens acetylcholine receptors modulate dopamine and motivation. Neuropsychopharmacology. 2016;41(12):2830-2838.

33. Foerde K, Steinglass JE, Shohamy D, Walsh BT. Neural mechanisms supporting maladaptive food choices in anorexia nervosa. Nat Neurosci. 2015;18(11):1571-1573.

34. Moore CF, Sabino V, Koob GF, Cottone P. Pathological overeating: emerging evidence for a compulsivity construct. Neuropsychopharmacology. 2017;42(7):1375-1389.

35. Yasoshima Y, Shimura T. A mouse model for binge-like sucrose overconsumption: contribution of enhanced motivation for sweetener consumption. Physiol Behav. 2015;138:154-164.

36. Klenotich SJ, Dulawa SC. The activity-based anorexia mouse model. Methods Mol Biol. 2012;829:377-393.

37. Klanker M, Feenstra M, Denys D. Dopaminergic control of cognitive flexibility in humans and animals. Front Neurosci. 2013;7:201.

38. Sanghvi IS, Singer G, Friedman E, Gershon S. Anorexigenic effects of $\mathrm{D}$-amphetamine and L-DOPA in the rat. Pharmacol Biochem Behav. 1975;3(1):81-86.

39. de Castro BM, et al. Reduced expression of the vesicular acetylcholine transporter causes learning deficits in mice. Genes Brain Behav. 2009;8(1):23-35.

40. Trudeau LE, El Mestikawy S. Glutamate cotransmission in cholinergic, GABAergic and monoamine systems: contrasts and commonalities. Front Neural Circuits. 2018;12:113.

41. Zhang H, Sulzer D. Regulation of striatal dopamine release by presynaptic auto- and heteroreceptors. Basal Ganglia. 2012;2(1):5-13.

42. Brimblecombe KR, Threlfell S, Dautan D, Kosillo $\mathrm{P}$, Mena-Segovia J, Cragg SJ. Targeted activation of cholinergic interneurons accounts for the modulation of dopamine by striatal nicotinic receptors. eNeuro. 2018;5(5):ENEURO.0397-17.2018.

43. Burguière E, Monteiro P, Feng G, Graybiel AM. Optogenetic stimulation of lateral orbitofronto-striatal pathway suppresses compulsive behaviors. Science. 2013;340(6137):1243-1246.

44. Steding J, et al. Goal-directed vs. habitual instrumental behavior during reward processing in anorexia nervosa: an fMRI study. Sci Rep. 2019;9(1):13529.

45. Kaye WH, Wierenga CE, Bailer UF, Simmons AN, Bischoff-Grethe A. Nothing tastes as good as skinny feels: the neurobiology of anorexia nervosa. Trends Neurosci. 2013;36(2):110-120.

46. Guzman MS, De Jaeger X, Drangova M, Prado MA, Gros R, Prado VF. Mice with selective elimination of striatal acetylcholine release are lean, show altered energy homeostasis and changed sleep/ wake cycle. J Neurochem. 2013;124(5):658-669.

47. Steinglass JE, Glasofer DR, Dalack M, Attia E. Between wellness, relapse, and remission: Stages of illness in anorexia nervosa. Int JEat Disord. 2020;53(7):1088-1096

48. Davis L, Walsh BT, Schebendach J, Glasofer DR, Steinglass JE. Habits are stronger with longer duration of illness and greater severity in anorexia nervosa. Int J Eat Disord. 2020;53(5):413-419.

49. Davis C, Carter JC. Compulsive overeating as an addiction disorder. A review of theory and evidence. Appetite. 2009;53(1):1-8.
50. Wills AM, Li R, Pérez A, Ren X, Boyd J, NINDS NET-PD Investigators. Predictors of weight loss in early treated Parkinson's disease from the NET-PD LS-1 cohort. J Neurol. 2017;264(8):1746-1753.

51. Lozano RH, Jofre IS. Novel use of L-dOPA in the treatment of anorexia and asthenia associated with cancer. Palliat Med. 2002;16(6):548.

52. Johnston JL, Leiter LA, Burrow GN, Garfinkel $\mathrm{PE}$, Anderson GH. Excretion of urinary catecholamine metabolites in anorexia nervosa: effect of body composition and energy intake. Am JClin Nutr. 1984;40(5):1001-1006.

53. Floresco SB. Prefrontal dopamine and behavioral flexibility: shifting from an "inverted-U" toward a family of functions. Front Neurosci. 2013;7:62.

54. Halabe Bucay A. Donepezil (aricept) as a treatment for anorexia nervosa: a very feasible therapeutic possibility. Expert Opin Investig Drugs. 2009;18(5):569-571.

55. Cummings JL, McRae T, Zhang R, Donepezil-Sertraline Study Group. Effects of donepezil on neuropsychiatric symptoms in patients with dementia and severe behavioral disorders. $A m \mathrm{~J}$ Geriatr Psychiatry. 2006;14(7):605-612.

56. Uwano C, et al. Rivastigmine dermal patch solves eating problems in an individual with advanced Alzheimer's disease. J Am Geriatr Soc. 2012;60(10):1979-1980.

57. Fassino S, et al. Prolonged treatment with glycerophosphocholine, an acetylcholine precursor, does not disclose the potentiating effect of cholinesterase inhibitors on GHRH-induced somatotroph secretion in anorexia nervosa. JEndocrinol Invest. 2003;26(6):503-507.

58. Kovacs J, et al. [Achetylcholinesterase (AChE) inhibition and serum lipokines in Alzheimer's disease: friend or foe?]. Neuropsychopharmacol Hung. 2012;14(1):19-27.

59. Bergman J, Miodownik C, Lerner PP, Miodownik E, Shulkin A, Lerner V. Donepezil as add-on treatment for resistant obsessive-compulsive disorder: retrospective case series. Clin Neuropharmacol. 2016;39(4):194-196.

60. Cubo E, Fernández Jaén A, Moreno C, Anaya B, González M, Kompoliti K. Donepezil use in children and adolescents with tics and attention-deficit/hyperactivity disorder: an 18-week, single-center, dose-escalating, prospective, open-label study. Clin Ther. 2008;30(1):182-189.

61. Albin RL, Minderovic C, Koeppe RA. Normal striatal vesicular acetylcholine transporter expression in Tourette syndrome. eNeuro. 2017;4(4):ENEURO.0178-17.2017.

62. Woon EP, Sequeira MK, Barbee BR, Gourley SL. Involvement of the rodent prelimbic and medial orbitofrontal cortices in goal-directed action: a brief review. JNeurosci Res. 2020;98(6):1020-1030.

63. Dautan D, et al. Cholinergic midbrain afferents modulate striatal circuits and shape encoding of action strategies. Nat Commun. 2020;11(1):1739.

64. Janickova H, et al. Deletion of the vesicular acetylcholine transporter from pedunculopontine/ laterodorsal tegmental neurons modifies gait. J Neurochem. 2017;140(5):787-798.

65. Kolisnyk B, et al. ChAT-ChR2-EYFP mice have enhanced motor endurance but show deficits in attention and several additional cognitive domains. J Neurosci. 2013;33(25):10427-10438. 